\title{
In vivo gene delivery of urokinase-type plasminogen activator with regulatable lentivirus induces behavioural changes in chronic cocaine administration
}

\author{
Amine Bahi, ${ }^{1}$ Frederic Boyer, ${ }^{1}$ Christèle Gumy, ${ }^{1}$ Tal Kafri ${ }^{2}$ and Jean-Luc Dreyer ${ }^{1}$ \\ ${ }^{1}$ Institute of Biochemistry, University of Fribourg, Rue du Musée 5, CH-1700 Fribourg, Switzerland \\ ${ }^{2}$ Gene Therapy Center and the Department of Microbiology and Immunology, University of North Carolina at Chapel Hill, Chapel \\ Hill, NC 27599-7352, USA \\ Keywords: addiction, drugs of abuse, lentivirus plasticity, rat, serine proteases
}

\begin{abstract}
Serine proteases play a key function in extracellular processes affecting central nervous system plasticity. Recently, the role of extracellular proteolytic processes in regulating synaptic structure and function has been described. However, to date direct evidence linking extracellular serine protease activity with drug-related behavioural changes has not been documented. Importantly, in a screening for genes induced after drug treatment we found that urokinase plasminogen-type activator (UPA) was strongly regulated by cocaine in several protocols of drug administration. Cocaine-induced up-regulation could be verified on microarray analysis under several protocols of drug administration, then further fully confirmed by means of qRT-PCR. As a result, we chose to investigate further UPA function in the mesolimbic dopaminergic pathway, a major target area of cocaine and drugs of misuse. Our approach was based on the characterization of cocaine-induced behavioural changes following lentiviral vector delivery of a doxycycline-regulated uPA expression cassette (or of its mutated form), into specific rat brain areas (the hippocampus, the nucleus accumbens and the ventral tegmental area). We show that doxycycline-dependent over-expression of uPA in these regions yields a 10- to 12.3-fold increase in locomotor activity after cocaine administration. These behavioural effects were completely abolished when the active site of the protease was point-mutated and used as a dominant negative. The physiological relevance of these drastic behavioural changes is discussed.
\end{abstract}

\section{Introduction}

Memory and learning as well as adaptive reorganizations after nervous system injury critically depend on synaptic plasticity, i.e. the capacity of synapses to change their structure and function to satisfy particular functional or adaptive requirements. Recently the role of extracellular proteolytic processes at the synapse for the regulation of synaptic structure, function and modification has been described (Gottschalk \& Deb, 1996; Lüthi et al., 1997; Shimizu et al., 1998; Chan \& Mattson, 1999; Komai et al., 2000; Shiosaka \& Yoshida, 2000). Serine proteases (e.g. neurotrypsin and tissue-type plasminogen activator) or inhibitors (such as neuroserpin) are considered to be involved in plasticity-related events in the nervous system (Yong et al., 1998).

In a screening for genes induced after drug treatment, we found that urokinase plasminogen-type activator (uPA) was strongly regulated by cocaine in several protocols. UPA is an inducible secreted serine protease found in vertebrates that specifically converts the inactive zymogen plasminogen into plasmin, a trypsin-like protease of broad substrate specificity (Appella et al., 1981). The plasminogen activation system has been implicated in multiple events requiring extracellular proteolysis in blood and tissues, such as blood clot dissolution, cell migration and invasion, inflammation, tissue repair and remodelling, and embryonic development (Gingrich \& Traynelis, 2000; Schwab

Correspondence: Dr J.-L. Dreyer, as above.

E-mail: jean-luc.dreyer@unifr.ch

Received 6 May 2004, revised 1 September 2004, accepted 20 September 2004 et al., 2001; Mustjoki, 2003). uPA is continuously produced in some tissues or cell types and only transiently in others, where it can be modulated by diverse effectors such as hormones, cytokines and growth factors. Accordingly, the uPA gene includes consensus motifs for the binding of a relatively large group of transcription factors involved in the transcriptional activation of both inducible and constitutively expressed genes (Nienaber et al., 2000). uPA associates with its functional urokinase receptor into a multifunctional system, involved in tissue remodelling, affecting cell migration and adhesion and plays a role in vitro nectin- and integrin-mediated cytoskeleton reorganization (Wang et al., 1998; Wang et al., 2000; Degryse et al., 2001). In human vascular smooth muscle cells, the complex is associated with the ecto-protein kinase casein kinase-2 (CK2), which, via phosphorylation of nucleolin, mediates mitogenic effects of urokinase and regulates urokinase-dependent cell adhesion to vitronectin (Stepanova et al., 2002).

uPA activity in the brain has been described mainly during development and is involved in axonal growth or tissue remodelling associated with neural development (Sumi et al., 1992; Mustjoki, 2003) and in neoplastic and cultured brain cells (Del Bigio et al., 1999). uPA, its receptor and its inhibitors are expressed in immature glial and neuronal cells in postnatal mouse forebrain. In the adult mice brain, uPA mRNA is not abundant but is strongly expressed by many classes of neurons in the peripheral and central nervous system and in spinal chord and sensory ganglia (Masos \& Miskin, 1996; Miskin \& Masos, 1997). It is localized in neurons in a few specific areas, 
primarily the subiculum and entorhinal cortex - which anatomically and functionally are linked to the hippocampus - and it is also found in the parietal cortex (Masos \& Miskin, 1996; Del Bigio et al., 1999). uPA mRNA increases substantially in the neocortex and limbic structures upon neuronal activation by kainic acid, a seizure-inducing agent (Masos \& Miskin, 1997), and uPA may be involved in hippocampal epileptogenesis (Kishi et al., 1999) as well as in brain tumours (Arai et al., 1998) or blood-brain barrier damage (Nienaber et al., 2000; Rosenberg et al., 2001).

In this study, we aimed to investigate the physiological function of uPA in the mesolimbic dopaminergic pathway, a major target area of cocaine and drugs of misuse. For this purpose a tetracyclineregulatable lentivirus, expressing uPA (or its mutated, inactive form) under the control of doxycycline, was prepared. After stereotaxic injection of the virus into specific rat brain areas, local expression of uPA from the lentivirus could be assessed and correlated with massive behavioural changes induced upon drug treatment. This approach enables us to switch locally gene expression on/off and to evaluate in the same animal how in vivo modulation affects drug-related behaviour. Drug addiction is a complex neuropsychiatric disorder, related to aberrant brain function, resulting in a loss of control over drug-taking or compulsive drug-seeking, despite noxious consequences (Nestler, 2000). Our approach may prove useful for understanding the molecular basis of this disorder, which requires the identification of molecular candidates and suitable animal models - to investigate the role of specific genes in mediating the development of drug addiction.

\section{Experimental procedures}

\section{Lentivirus construction of Lenti-uPA-His6 and Lenti-GFP}

The rat uPA cDNA (GenBank accession no. X63434) was amplified by reverse transcription. Briefly, $2 \mu \mathrm{g}$ of total RNA [prepared from rat ventral tegmentum area (VTA) of cocaine-treated animals] was added to $1 \mu \mathrm{g}$ Oligo- $(\mathrm{dT})_{12-18}, 2 \mu \mathrm{L}$ of dNTP mix at $10 \mu \mathrm{M}$ each and made up to $12 \mu \mathrm{L}$ with Rnase-free water. These components were mixed and heated at $65{ }^{\circ} \mathrm{C}$ for $5 \mathrm{~min}$, then kept on ice. To the mixture, $4 \mu \mathrm{L}$ of $5 \times$ first-strand buffer was added, followed by $2 \mu \mathrm{L} 0.1$ M DTT, $10 \mathrm{U}$ RNAsin, and $1 \mu \mathrm{L}$ of $200 \mathrm{U} / \mu \mathrm{L}$ Superscript II RNaseH Reverse Transcriptase (Invitrogen, Basel, Switzerland). The mixture was incubated at $42{ }^{\circ} \mathrm{C}$ for $3 \mathrm{~h}$. To remove RNA-DNA hybrids, $2 \mathrm{U}$ RNase $(\mathrm{H})$ was added and incubated at $37{ }^{\circ} \mathrm{C}$ for $30 \mathrm{~min}$. The cDNA was then PCR amplified and 6His-tagged with the two following primers: CGCGGATCCGCATGAGAGTCTGGCTTGCG as forward primer and CCGCTCGAGCGGTTAATGATGATGATGATGATGA TTAAGTTAACACTGC as reverse primer. The forward primer contains a BamHI restriction site (bold) followed by the $5^{\prime}$ rat uPA cDNA-specific sequence, and the reverse primer contains the $3^{\prime}$ rat uPA cDNA-specific sequence, a 6His-Tag (underlined), a stop codon and an $X h o$ I restriction site (bold). The PCR product was digested with BamHI and XhoI and cloned into similar sites in pTK431 (Fig. 1A). The pTK431 is a self-inactivating (SIN) HIV-1 vector, which contains the entire tet-off inducible system, the cPPT and the woodchuck hepatitis virus post-transcriptional regulatory element (WPRE) (Bahi et al., 2004). It was generated by ligating a $B g l \mathrm{II} / \mathrm{BamHI}$ DNA fragment containing the tetracycline-regulated transactivator tTA $\left(5^{\prime}\right)$ and the tetracycline inducible promoter (3') into a BamHI site downstream to a CMV promoter in an SIN HIV-1 vector. A control vector construct, pTK433 (Fig. 1A), in which green fluorescent protein (GFP) expression is regulated by a tetracycline inducible promoter, was generated by cloning a BamHI/BglII DNA fragment containing the GFP gene into a BamHI site in pTK431 (Bahi et al., 2004). All plasmids were $\mathrm{CsCl}_{2}$ purified.

\section{Site-directed mutagenesis and construction of Lenti-uPA-Mut}

The uPA cDNA sequence was point-mutated in two active sitecomposing amino acids, $\mathrm{H} 225 \mathrm{G}(\mathrm{CAC} \rightarrow \mathrm{GGC})$ and S377A (TCA $\rightarrow$ GCA). Mutagenesis was performed with the QuickChange site-directed mutagenesis kit (Stratagene, Switzerland). The following oligonucleotides (single-stranded sense and antisense oligonucleotides) were used as primers; (H225 G): 5'-GGGTGGCCAGCGCCA CAggCTGCTTCGTGAATCAGCC- $3^{\prime}$ and $5^{\prime}$-GGCTGATTCACGAA GCAGccTGTGGCGCTGGCCACCC-3'; (S377A): 5'-GCTCGGGA GATgCAGGAGGACCTCTTATCTGTAACATCG-3' and CGATGTT ACAGATAAGAGGTCCTCCTGcATCTCCCGAGC. PCRs were performed with wild-type pCRII-uPA-His6 cDNA as template, following the manufacturer's instructions. The PCR products, digested by $D p n \mathrm{I}$ (Biolabs, Switzerland), were used for transformation and amplified. Mutations were verified by sequencing. The insert was then digested with $B a m \mathrm{HI}$ and $\mathrm{XhoI}$ and cloned into pTK431 transfer vector. This construct, pTK431-uPA-Mut, was used for triple transfection and preparation of lentivirus (Lenti-uPA-Mut).

\section{Lentivirus production}

The vector plasmid (either pTK431-uPA-6His, pTK431-uPA-Mut or pTK433), together with the packaging construct plasmid $\mathrm{p} \Delta \mathrm{NRF}$ and the envelope plasmid pMDG-VSVG were co-transfected into HEK293T cells to produce the Lenti-uPA-His6 or Lenti-GFP viral particles, respectively, according to previously published procedures (Naldini et al., 1996; Bahi et al., 2004). High-titre stocks were obtained by ultracentrifugation at $45000 \mathrm{~g}$ for $2 \mathrm{~h}$ at $4{ }^{\circ} \mathrm{C}$. The pellet was resuspended in PBS containing $1 \%$ bovine serum albumin, and stored frozen at $-80{ }^{\circ} \mathrm{C}$. The viral titres were determined by $\mathrm{p} 24$ antigen measurements (KPL, USA). For in vivo experiments, the different viral stocks matched for viral particle content were used at $0.2 \mathrm{mg} / \mathrm{mL}$ of $\mathrm{p} 24$, corresponding to $8 \times 10^{9}$ infectious particles $/ \mathrm{mL}$.

\section{Microarray design and analysis}

Microarray design was performed according to published methods (Shalon et al., 1996). Briefly, specific oligos were synthesized with 5 '-amino modification and arrayed onto QMT Aldehyde Slides (Quantifoil, Germany) and stored at room temperature for further hybridization.

Probe design was according to that of Bahi \& Dreyer (2004) and recommendations by QIAGEN ${ }^{\mathrm{TM}}$. Briefly, $70 \mathrm{mer}$ probes for ten negative and ten positive control genes and for uPA were designed within 1000 bases from the $3^{\prime}$ end of the available gene sequence; probes were selected with an optimal set of parameters, sequence optimized using BLAST for nucleotide sequence to minimize crosshybridization in microarray experiments and $T_{\mathrm{m}}$ (melting temperature) was normalized to $78^{\circ} \mathrm{C}\left( \pm 5^{\circ} \mathrm{C}\right)$.

The ten negative controls were oligonucleotides randomly generated with no expression within either the mouse or the rat genomes. The ten positive controls were oligonucleotides specific for $\beta$-actin (NM_031144), transcription factor E2a (X17500), lactate dehydrogenase A (NM_017025), Hsp70-1 (NM_031971), nuclear pore glycoprotein 62 (M62992), ribophorin 20814 (NM_031698), ribosomal protein L5 (NM_031099), ribosomal protein S9 (NM_031108), glyceraldehyde-3-phosphate dehydrogenase (GAPDH) 


\section{A. CONSTRUCTS}

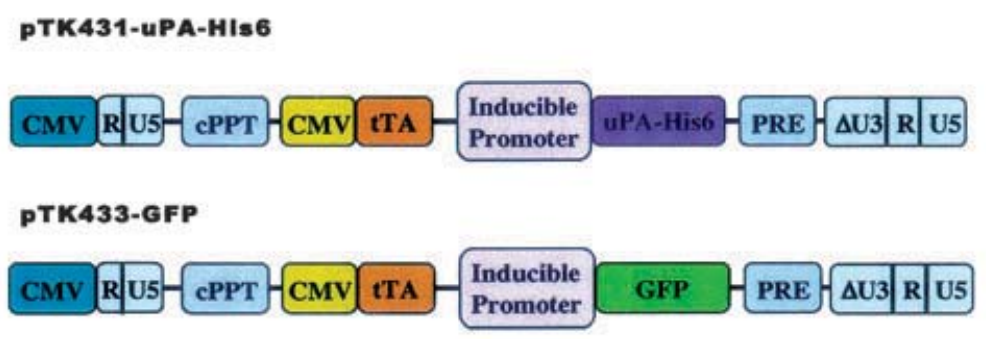

\section{B. DRUG ADMINISTRATION PROTOCOLS}

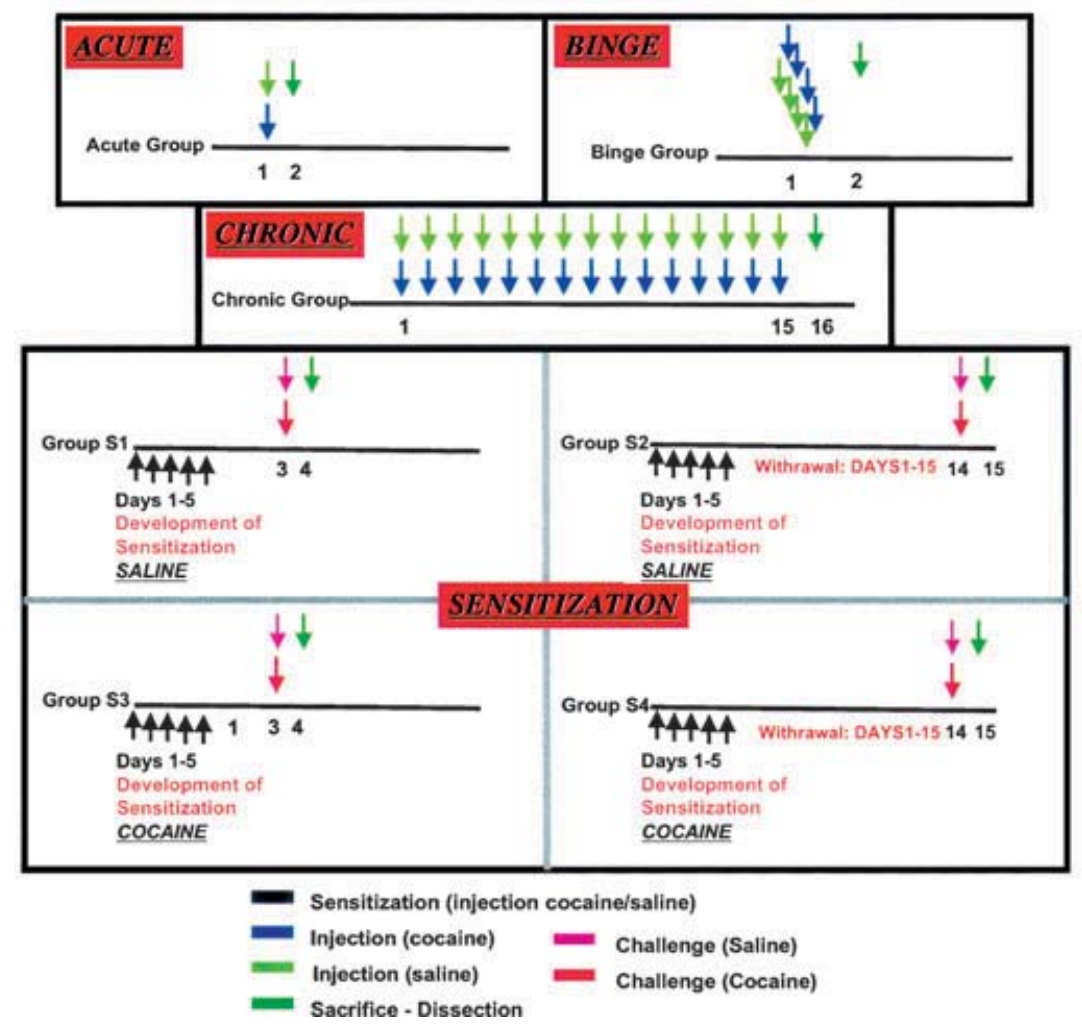

FIG. 1. (A) Lentivirus constructs. Transfer plasmids pTK431-uPA-6His and pTK433-GFP used in this study for the production of Lenti-uPA-6His and Lenti-GFP. The plasmids contain a Tet-Off element under the control of a minimal CMV promoter and a cPPT element. (B) Drug administration protocols. Acute treatment: animals $(n=6)$ were injected with a single dose of cocaine $(15 \mathrm{mg} / \mathrm{kg})$ and killed $24 \mathrm{~h}$ after injection. Binge treatment: animals $(n=6)$ received four injections of high-dose cocaine $(30 \mathrm{mg} / \mathrm{kg})$ and were killed $24 \mathrm{~h}$ after the last injection. Chronic treatment: animals $(n=6)$ received a single dose of cocaine $(15 \mathrm{mg} / \mathrm{kg})$ every day over 15 days and were killed $24 \mathrm{~h}$ after the last injection. 'Behavioural sensitization' treatment: four groups of animals $(n=6)$ were used. Initially each group received daily injections over 5 days of either saline (groups S1 and S2) or cocaine (15 mg/kg, groups S3 and S4). Three days after the last injection, groups S1 and S3 were challenged with a single dose of saline (group S1) or cocaine (15 mg/kg, group S3) and killed $24 \mathrm{~h}$ after the injection. Fourteen days after the last injection, groups S2 and S4 were challenged with a single dose of saline (group S2) or cocaine (15 mg/kg, group S4) and killed $24 \mathrm{~h}$ after the last injection.

(NM 017008) and polyubiquitin (D16554). Oligonucleotide sequences of positive and negative controls are patented (QIAGEN $^{\mathrm{TM}}$, Basel, Switzerland) and are not listed. For uPA the following oligonucleotide was used: 5'-TGCAAGTCTAGGTATTTCCCTCC CTCCAGACTGTGATGCGGCCCATTTGGTCTTCCGTGATGCTC CACTT-3'.

For probe preparation, each aRNA sample (one from control samples and one from treated samples) was reverse-transcribed in the presence of Cy3-dCTP (control samples) or Cy5-dCTP (treated samples) and purified, according to the methods of Bahi \& Dreyer (2004). Before hybridization, the probe solution was boiled for $1 \mathrm{~min}$, and then rapidly applied to the microarray under a cover slip. Slides were placed in hybridization chambers and incubated for $14-16 \mathrm{~h}$ in a water bath at $64{ }^{\circ} \mathrm{C}$, then washed and dried before scanning with a scanning laser microscope (Affymetrix ${ }^{\circledR} 428^{\mathrm{TM}}$ array scanner; Affymetrix, Inc., Santa Clara, CA, USA).

Separate images were acquired for each fluorochrome at a resolution of $10 \mu \mathrm{m}$ per pixel. To normalize the two channels with respect to signal intensity, photomultiplier and laser power settings were adjusted such that the signal ratio of the control genes was as close to 1.0 as possible. The average fluorescence intensity for each fluorochrome and for each gene was determined using Jaguar 2.0 
(Affymetrix), which calculates normalized expression levels and ratios of experimental (Cy5) vs. control (Cy3) signals. Background fluorescence was calculated as the median fluorescence signal of non-target pixels around each gene spot. The means of values of the negative controls were considered as background and subtracted from all other data. Positive controls displayed neither up- nor down-regulation in all samples under investigation and the ratio of experimental vs. control was almost equal to one in all cases.

Data sets were further statistically analysed and $P$-values evaluated using a stringent cut-off for significance. Induction or repression of a gene was defined as a minimum 1.5-fold change in its transcript level. Genes were considered to be down-regulated if the ratio of experimental to control was $0.12-0.5$ (or -0.12 to -0.5 ) with $P<0.01$ or up-regulated if the ratio of experimental to control was 2 or more with $P<0.01$. Significance was calculated using a $t$-test.

\section{Drug administration protocols for microarray analysis and quantitative real-time PCR}

Animals used in this experiment were male Wistar rats weighing 225$250 \mathrm{~g}$ (BRL, Füllinsdorf, Switzerland). All animal experiments were carried out in accordance with the guidelines and regulations for Animal Experimentation, BAG, Bern, Switzerland. The animals were housed in threes in clear plastic cages with wire grid lids. Access to food and water was unrestricted. The animals were kept in the animal facility maintained on a 12-h light : 12-h dark cycle (lights off at 07:00 h). Four different protocols for drug administration were used, as described in Fig. 1B.

1 Acute Protocol: animals $(n=6)$ were i.p. injected once with

$15 \mathrm{mg} / \mathrm{kg}$ cocaine $/ \mathrm{HCl}$ (Sigma Chemical Co.).

2 Chronic Protocol: animals $(n=6)$ were i.p. injected daily with

$15 \mathrm{mg} / \mathrm{kg}$ cocaine $/ \mathrm{HCl}$ for a period of 15 days;

3 Binge Protocol: animals $(n=6)$ were injected i.p. in total four times with $30 \mathrm{mg} / \mathrm{kg}$ cocaine $/ \mathrm{HCl}$ every $2 \mathrm{~h}$.

In all three protocols control animals received a $0.9 \%$ saline i.p. injection instead of drug under the same schedule. Twenty-four hours after the last injection, animals were killed by decapitation and the brain area were dissected out and used for isolation of total RNA, using RiboPure Kit (Ambion, UK) followed by RNA amplification according to previous methods (Kacharmina et al., 1999; Bahi et al., 2004).

4 Sensitization Protocol: four groups ( $n=6$ per group) were used, designated groups S1-S4. Each group was treated with either saline or cocaine for 5 days, then challenged with either saline or cocaine 3 or 14 days after the last injection, and finally killed $24 \mathrm{~h}$ later after the challenge by decapitation and the brain area were dissected out and used for isolation of total RNA followed by RNA amplification.

\section{Groups S1 and S2}

Animals were treated with daily i.p. injection of $0.9 \%$ saline over a period of 5 days. Group S1 was challenged 3 days after the last injection with i.p. injection of $0.9 \%$ saline, and group S2 was challenged 14 days after the last injection with i.p. injection of $15 \mathrm{mg} / \mathrm{kg}$ cocaine/ $\mathrm{HCl}$.

\section{Groups S3 and S4}

Animals were given daily i.p. injection of $15 \mathrm{mg} / \mathrm{kg}$ cocaine $/ \mathrm{HCl}$ (Sigma Chemical Co.) over a period of 5 days. Three days after the last injection group S3 was challenged with i.p. injection of $0.9 \%$ saline daily, and 14 days after the last injection group S4 was challenged with i.p. injection of $15 \mathrm{mg} / \mathrm{kg}$ cocaine $/ \mathrm{HCl}$.
For real-time PCR analysis, total RNA was not amplified before PCR performing. See 'In vitro assays' for further details concerning the methodology and oligos used in this study.

\section{Strereotaxic surgery and behavioural analysis}

Stereotaxic surgery and injection of the lentiviral vector were performed according to previously described methods (Bahi et al., 2004). Male Wistar rats were bilaterally injected into either the VTA, the NAcc or into the hippocampus with $2 \mu \mathrm{L}$ of concentrated lentiviral stock $\left(0.2 \mathrm{mg} / \mathrm{mL}\right.$ of $\mathrm{p} 24$, corresponding to $\left.8 \times 10^{9} \mathrm{IU} / \mathrm{mL}\right)$. The injections were performed bilaterally at the following coordinates, as calculated from bregma and the dura mera: (a) in the VTA: anterior -6 ; lateral \pm 0.6 ; ventral -8 ; (b) in the NAcc: anterior +1.4 ; lateral \pm 1.6 ; ventral -6.8 ; (c) in the ventral subiculum of the hippocampus: at two different coordinates, designated VSub-1 and VSub-2, with VSub-1: anterior -5.3 ; lateral \pm 4.8 ; ventral -6.8 ; and VSub-2: anterior -6 ; lateral \pm 5.3 ; ventral -4 . Following surgery the diet consisted of normal food pellets soaked in water for 3 days.

One week after surgery, locomotor activity was monitored in MEDOFA-RS cages (MED Associates Inc., USA) during the dark cycle in daily sessions over 16 days, according to previously published procedures (Bahi et al., 2004). The animal was placed in the activity-monitoring cage for a 30-min baseline recording without drug. The session was then automatically paused and each subject received cocaine $/ \mathrm{HCl}(15 \mathrm{mg} / \mathrm{kg}$, i.p) and was then placed back into the locomotor activity-monitoring cage for a further $60 \mathrm{~min}$.

Where assessing dose-dependent effects of cocaine on uPAmediated changes in locomotor activity, animals were injected with Lenti-uPA-His6 into the NAcc using the same coordinates as above and were administered a single i.p. injection of $7,10,15$ or $30 \mathrm{mg} / \mathrm{kg}$ of cocaine $/ \mathrm{HCl}$.

The activity monitor computed the location of the animal in each of the $\mathrm{X}$ and $\mathrm{Y}$ dimensions as the middle point between the extreme beam interruptions. Speed was estimated by computing standard deviation of the distances of data points to their mean within a sliding time window $0.4 \mathrm{~s}$ wide. Within the same experimental group, means and standard deviations were recorded for measurements performed each day during one session. The $t$-test was performed by comparison of the six animals (three from each group) at the same time point and the $P$-value was calculated to check for the significance of the difference between the groups with and without doxycycline.

\section{Immunohistochemistry}

Rat brains were rapidly removed and frozen in isopentane (at $-30{ }^{\circ} \mathrm{C}$ for $3 \mathrm{~min}$ ) and kept at $-25^{\circ} \mathrm{C}$. Coronal sections were cut at $14 \mu \mathrm{m}$ in a cryostat (Leitz), placed on gelatinized glass slides, air-dried at room temperature for $20 \mathrm{~min}$ and kept at $-25{ }^{\circ} \mathrm{C}$ until further processing. Antigens were localized using the avidin-biotin-peroxidase technique.

Slices were fixed in 4\% PFA for $15 \mathrm{~min}$, and washed three times with $1 \times$ PBS. Endogenous peroxidase activity was quenched with $2 \%$ hydrogen peroxide in water $\left(\mathrm{H}_{2} \mathrm{O}_{2}\right)$ for $40 \mathrm{~min}$ at room temperature (RT). Non-specific binding was blocked for $30 \mathrm{~min}$ at RT in $1 \times$ PBS containing $1 \%$ bovine serum albumin, $1 \%$ Triton $\mathrm{X}-100$ and $3 \%$ normal goat serum. Sections were then rinsed and incubated overnight with mouse anti-histidine antibody (MCA1396, Serotec, $1: 12$ 000) diluted in $1 \times$ PBS containing $1 \%$ Triton X-100 and $1 \%$ normal goat serum. Sections were then washed three times with $1 \times$ PBS and incubated with the biotinylated secondary antibody (goat anti-mouse immunoglobulin G, Vector Laboratories, Burlingame, CA, USA, 
$1: 500$ ) for $45 \mathrm{~min}$ at RT. Sections were rinsed three times for $5 \mathrm{~min}$ in $1 \times$ PBS at RT, followed by avidin-biotin complex (Vector Laboratories) in $1 \times$ PBS solution. After three rinses in $1 \times$ PBS, all sections were developed in $0.025 \% 3-3^{\prime}$ diaminobenzidine tetrahydrochloride (DAB) plus $0.02 \% \mathrm{H}_{2} \mathrm{O}_{2}$ for $10-15 \mathrm{~min}$. Sections were then dehydrated, mounted in permanent medium (Eukitt) and examined with a Zeiss light microscope. GFP-expressing sections were cover-slipped with AF1 mounting solution (Citifluor Ltd), and directly observed using a multifluorescence Axioplan 2 imaging microscope (Zeiss, Germany) mounted with a multichannel Axiocam camera (Zeiss, Germany) combined with Axiovision 3.1 acquisition software (Zeiss, Germany). In all cases negative controls included omission or substitution of the primary antibody.

\section{In vitro assays}

The efficiency of the Lenti-uPA-His6 was tested in vitro by infection of HEK293T cells: $3 \times 10^{5}$ HEK293T cells were plated per well in six-well plates. The next day, $1-4 \mu \mathrm{L}$ from lentiviruses stocks was mixed with $10 \mu \mathrm{g} / \mathrm{mL}$ Polybrene (Sigma, Buchs, Switzerland) with/without $30 \mathrm{ng} / \mathrm{mL}$ doxycycline (Sigma, Buchs, Switzerland), incubated for $30 \mathrm{~min}$ at RT, added to the cells and incubated at $37{ }^{\circ} \mathrm{C}$. After $24 \mathrm{~h}$ the medium was replaced with normal growth medium and cells were left for a further $24 \mathrm{~h}$. One portion of the cells was used for total RNA isolation (for real-time PCR) and the remaining part was used for immunocytochemistry.

Quantitative real-time PCR and quantification of $u P A m R N A$

For quantitative real-time PCR (qRT-PCR), primer sets for rat uPA, GAPDH and $\beta$-actin were designed to amplify 100 - to $300-b p$ products, using PRIMER3 software (http://frodo.wi.mit.edu/cgi-bin/ primer3/primer3_http://www.cgi). The following specific primer pairs were used:

for rat uPA,

5'-CAGTCCTTGCGTGTCTGCC-3' and

5'-CGCTGGGCTCTCTCTAGACAG-3';

for rat GAPDH,

5'-ATGACTCTACCCACGGCAAG-3' and

5'-CATACTCAGCACCAGCATCAC-3';

for rat $\beta$-actin,

5'-AGCCATGTACGTAGCCATCC-3' and

5'-CTCTCAGCTGTGGTGGTGAA-3'.

GAPDH and $\beta$-actin were used as endogenous controls for normalization. No difference was observed between these two genes for all quantifications.

Total RNA was extracted from the HEK293T cells using RiboPure Kit (Ambion, UK) including a RNase-free DNase step, and stored at $-80{ }^{\circ} \mathrm{C}$. RNA was quantified by spectrophotometry, and its integrity verified by agarose gel electrophoresis as visualized with ethidium bromide staining. First-strand cDNA was generated from $1 \mu \mathrm{g}$ of total RNA and Oligo $\left(\mathrm{dT}_{12-18}\right)$ primer with the M-MLV reverse transcription kit (Invitrogen) in a total volume of $20 \mu \mathrm{L}$. The reaction product was used for qRT-PCR using the real-time PCR iCycler (Bio-Rad, Reinach, Switzerland). Five microlitres of cDNA preparation, $0.5 \mu \mathrm{M}$ of forward and reverse primers and $10 \mu \mathrm{L}$ of IQ SYBR Green Supermix (Bio-Rad) in a total volume of $20 \mu \mathrm{L}$ were applied and PCR was performed as follows: $3 \mathrm{~min}$ at $95^{\circ} \mathrm{C}$ (initial denaturation); $20{ }^{\circ} \mathrm{C} / \mathrm{s}$ temperature transition rate up to $95{ }^{\circ} \mathrm{C}$ for $45 \mathrm{~s}, 45 \mathrm{~s}$ at $62{ }^{\circ} \mathrm{C}$, repeated 40 times (amplification). The PCR reaction was evaluated by melting curve analysis and by checking the PCR products on $2 \%$ agarose gel.
The PCR cycle number at which each assay target reached the threshold detection line was determined ( $\mathrm{Ct}$ value). cDNA samples were assayed on at least three dilutions to check for assay reliability using a duplicate assay on each dilution. The $\Delta \mathrm{Ct}$ for each candidate was calculated as $\Delta \mathrm{Ct}=[\mathrm{Ct}$ (candidate) $-\mathrm{Ct}$ (GAPDH or $\beta$-actin) $]$. The relative abundance of each target in each protocol can be calculated as the ratio between treated and untreated samples (Mühlbauer et al., 2004).

\section{Immunocytochemistry on HEK293T cells}

At the end of the infection period, cells were washed twice with $1 \times$ PBS, fixed with prewarmed $4 \%$ PFA for $30 \mathrm{~min}$ and washed again with $1 \times$ PBS buffer. Cells were blocked for non-specific binding with $3 \%$ normal goat serum diluted in $1 \times$ PBS for $20 \mathrm{~min}$, washed twice with the same buffer and incubated for $3 \mathrm{~h}$ at RT with mouse anti-histidine-tag antibody (MCA1396, Serotec, $1: 200$ ) in $1 \times$ PBS containing $1 \%$ normal goat serum and $0.1 \%$ Triton-X100. Cells were rinsed three times with $1 \times$ PBS and incubated with the secondary antibody (FITC-conjugated goat anti-mouse immunoglobulin G, Molecular Probes, $1: 1000$ ) in 1\% normal goat serum, 0.1\% Triton-X100 in the dark at RT for $3 \mathrm{~h}$. Cells were washed three times with $1 \times$ PBS and cover slipped with AF1 mounting solution (Citifluor Ltd). In all cases negative controls included omission or substitution of the primary antibody. Stained cells were observed using a multi-fluorescence Axioplan 2 imaging microscope (Zeiss, Germany) mounted with a multi-channel Axiocam camera (Zeiss, Germany) combined with Axiovision 3.1 acquisition software (Zeiss, Germany). The fluorophore was detected with the appropriate detecting system.

\section{Results \\ uPA up-regulation in different drug administration protocols}

uPA was found initially in a screening for genes up-regulated after cocaine administration. In order to characterize this observation further, up-regulation of uPA was confirmed in different protocols of drug administration, summarized in Fig. 1B, i.e. acute, binge, chronic and four additional treatments ('behavioural sensitization'). The acute protocol corresponds to a single exposure to the drug, as opposed to the chronic treatment, which was used in all behavioural assays. The binge protocol mimics the treatments in humans and allows for easy assessments of neurobiological changes associated with addiction (Michna et al., 2002). The fourth protocol is an established model of 'behavioural sensitization' (Heidbreder et al., 1996), subdivided into two distinct temporal parts, initiation and expression; based on this schedule, data have shown that some genes are involved in the latter, but not in the former phase (Hedou et al., 2002).

uPA-specific oligonucleotides were spotted on a glass array, together with positive and negative controls (see Methods). The total RNA from four different brain regions of animals treated under the different protocols (Fig. 1B, $n=6$ ) was prepared and hybridized. As shown in Fig. 2A, uPA was up-regulated in most treatments and in all regions investigated. In the hippocampus, the up-regulation was strongest in S3 and S4 groups (i.e. animals treated with cocaine over 5 days and challenged with the drug either after 3 days or after 2 weeks of withdrawal, respectively), whereas a two-fold up-regulation was observed in this region after chronic treatment. In the NAcc, up-regulation was very strong under all treatments: in groups S3 and S4 of the 'behavioural sensitization' protocol or after chronic treatment, a 5- to 5.8-fold up-regulation was observed, whereas under other treatments, we found a $c a$. four-fold up-regulation, except for 
group S1 (animals initially treated with saline and challenged with saline after 3 days). In the striatum (caudate putamen) the effects were similar but weaker, yielding up to five-fold up-regulation (group S4).
Finally in the VTA very strong up-regulation was observed in group S4 ( $>$ six-fold) and S3, or in binge (four-fold increase in both groups). In all cases the increase was highly significant $(P<0.01)$. Other brain

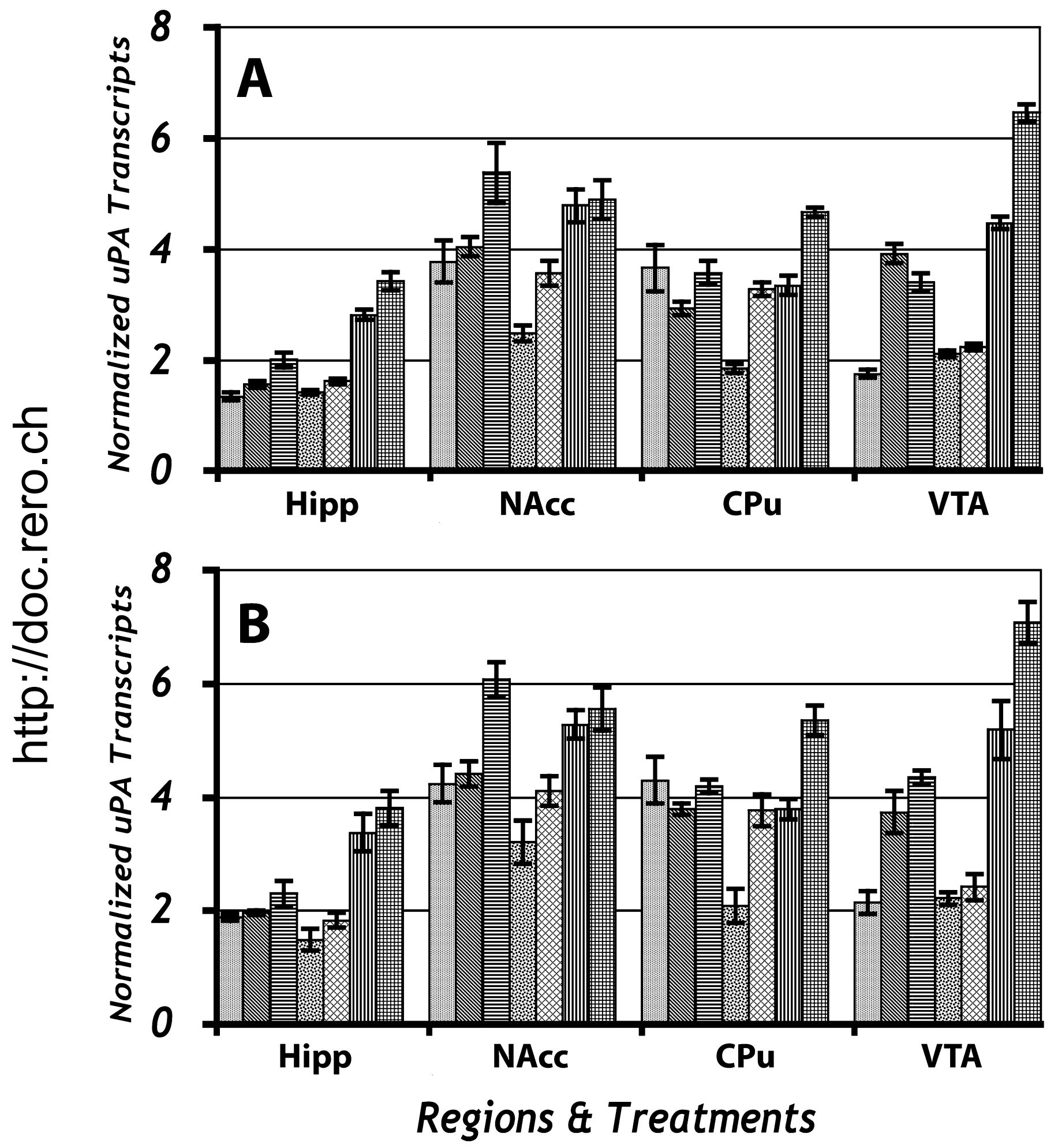

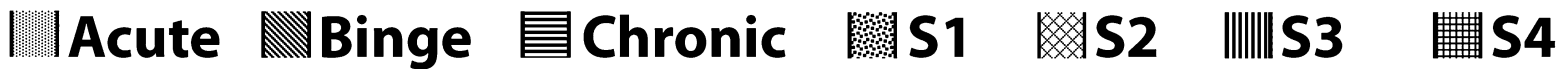

FIG. 2. 
regions have not been tested. Under these conditions all control genes are unaffected.

In order to verify and confirm these data from microarray analysis, the drug administration protocols were replicated on another set of animals $(n=6)$ treated under the same protocols, and total RNA from the four brain regions were prepared and used for real-time quantitative PCR. All results from microarray analysis could be fully confirmed by means of qRT-PCR in all four brain areas using uPAspecific oligos, as shown in Fig. 2B. In all cases the changes are again highly significant $(P<0.01)$.
Following repeated cocaine administrations ('chronic' and 'behavioural sensitization' protocols) uPA mRNA is up-regulated. This overexpression is much higher after 14 days of withrawal. mRNA levels before the challenge times have not been measured (as no animal were killed just prior to the challenge time); however, the data presented in this study are from samples of animals killed $24 \mathrm{~h}$ after the last drug administration and therefore the levels of mRNA are relatively stable for at least $24 \mathrm{~h} \mathrm{(e.g.} \mathrm{in} \mathrm{the} \mathrm{NAcc} \mathrm{six-fold}$ increase, in the VTA four-fold increase, vs. saline, respectively). By contrast, even after 3 days of drug removal (group S3) the mRNA

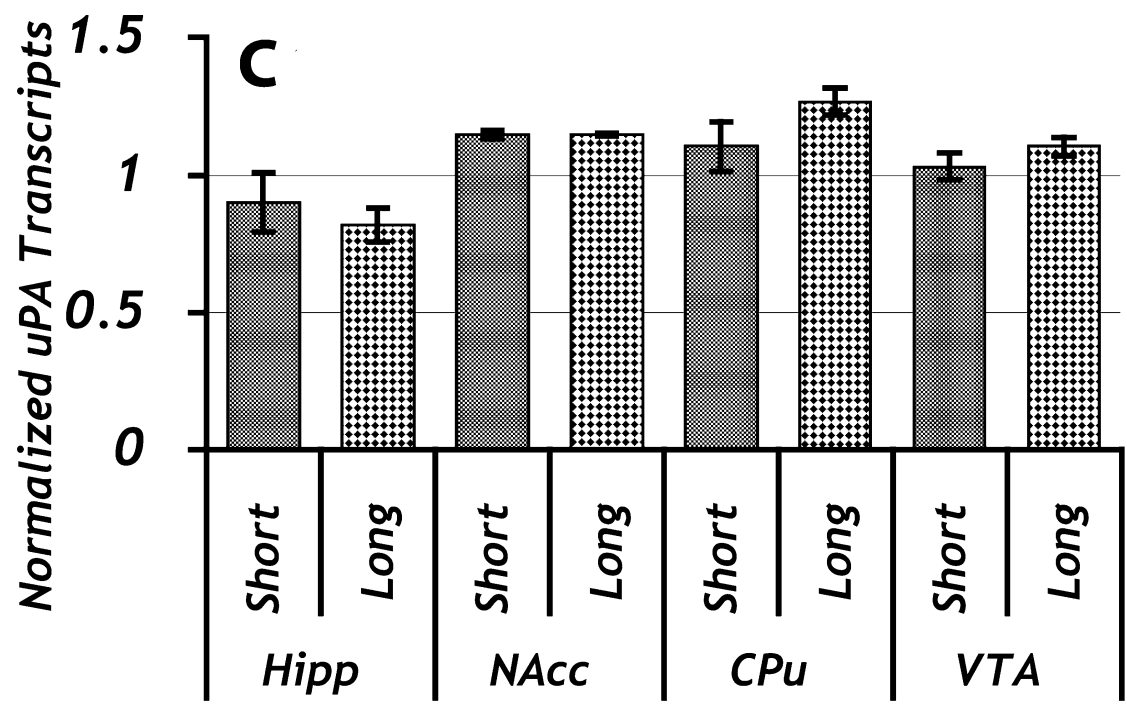

\section{Comparing sensitization schedules S1 - S4}

\begin{tabular}{lcccc}
\hline & Hipp & NAcc & CPu & VTA \\
\hline S1 Vs S3 & $0.035^{*}$ & $0.024^{*}$ & $0.026^{*}$ & $0.015^{*}$ \\
\hline S2 Vs S4 & $0.013^{*}$ & $0.047^{*}$ & $0.028^{*}$ & $0.004^{* *}$ \\
\hline \multicolumn{6}{c}{$P<0.05,{ }^{*} P<0.01$}
\end{tabular}

FIG. 2. Microarray and qRT-PCR analysis of uPA expression changes of cocaine-treated animals. (A) Microarray analysis of uPA expression changes in different brain regions and different drug delivery protocols. Microarrays were prepared as microscope slides as described in the Methods section from specific 70mer oligos. Candidates were spotted eight times on a glass microarray, together with controls. Controls included 20 candidates (ten positive and ten negative controls, see Methods). Animals ( $n=6$ ) were injected with cocaine according to the different protocols (Fig. 1). Total RNA from four brain areas, including the tegmentum, the NAcc, the hippocampus and the dorsal striatum of cocaine-treated and saline-treated animals were prepared, labelled with Cy5-dCTP (cocaine-treated subjects) and Cy3-dCTP (saline-treated subjects) and hybridized on the microarrays. aRNA preparation and hybridization was performed from three independent tissue samples of cocaine- or saline-treated animals. Arrays were scanned and analysed as described. (B) qRT-PCR of cocaine-induced uPA transcript changes. Animals ( $n=6$ ) were treated under the same drug administration protocols as in A, killed and dissected. Total RNA from the four brain areas were prepared and reverse transcribed for qRT-PCR using uPA specific oligos (see Methods). (C) uPA transcript levels from saline-challenged animals in S3 and S4 schedules (see 'sensitization' protocols, Fig. 1B). After cocaine administration for 5 days followed by saline challenge, respectively 3 and 14 days after the last injection, mRNA levels of groups S3 (short withdrawal) and S4 (long withdrawal) were compared. Saline challenge had almost no effect on uPA expression and the ratio was equal to 1.0. (D) $P$-values of ratios of uPA expression during 'sensitization' schedules. uPA expression after initial cocaine delivery (S3 and S4 groups) compared with saline (S1 and S2 groups) was significantly higher, as shown by calculation of the $P$-values. Hipp: hippocampus, NAcc: nucleus accumbens, CPu: caudate putamen, VTA: ventral tegmentum area, Short: short withdrawal, Long: long withdrawal, $* P<0.05, * * P<0.01$. 
levels reached basal values (Fig. 2C). This indicates that mRNA is relatively stable for at least $24 \mathrm{~h}$ in the chronic protocol used in behavioural studies, in good correlation with our findings that locomotor activity changes were observed within the first $30 \mathrm{~min}$ prior to cocaine delivery (see below). In addition, it has been established that half-life of uPA in cells is about $2 \mathrm{~h}$ (Sokabe et al., 2004).

It is interesting to observe from the 'behavioural sensitization' protocols that, when initially treated with cocaine, animals express more uPA mRNA than when initially treated with saline (Fig. 2D). This overexpression is highly significant in all brain areas tested. Figure 2D illustrates the difference observed between the various treatments during the 'behavioural sensitization' protocols: S1 vs. S3 and $\mathrm{S} 2$ vs. $\mathrm{S} 4(* P<0.05, * * P<0.01)$.

A detailed analysis of microarray and qRT-PCR data further shows that UPA mRNA is not induced when animals were challenged with saline, even after repeated cocaine injections, and mRNA levels are not affected after a short or even long withdrawal period ( 3 vs. 14 days). As shown in Fig. $2 \mathrm{C}$, the ratios of uPA/GAPDH are almost equal to 1.0 in all cases. The same results were observed when normalization was performed against $\beta$-actin (data not shown). There seems to be no conditioned induction of uPA mRNA levels when rats are challenged with saline following repeated cocaine administrations.
In vitro assay of lentivirus-mediated uPA expression in HEK293T cells

uPA was cloned into a tetracycline-regulatable lentivirus system and the lentivirus expressing uPA (Lenti-uPA-His6) was used for in vitro assays (see Methods). HEK293T cells were infected with different amounts of virus (Fig. 3A) and uPA mRNA was measured by qRTPCR. As shown, Lenti-uPA-His6 increases the uPA transcripts and protein in a dose (titre)-dependent way. Using $1 \mu \mathrm{L}$ of the viral stock, uPA mRNA increases by 1.5 -fold; this reaches two-fold using $2 \mu \mathrm{L}$ of viruses. Finally, a four-fold increase in uPA mRNA was observed with $4 \mu \mathrm{L}$. This increase is completely reversible using doxycycline, which blocks uPA expression, both at the mRNA and at the protein levels (Fig. 3A and B). For real-time PCR, uPA transcripts levels were normalized against both GAPDH and $\beta$-actin used as endogenous controls. No difference was observed between these two genes (data not shown).

\section{Behavioural changes induced by local expression of wild-type and mutated forms of UPA using regulatable lentiviruses in vivo}

Lentivirus expressing either wild-type uPA (Lenti-uPA-His6), its mutated form (Lenti-uPA-Mut) or GFP (Lenti-GFP, as a control) was stereotaxically inoculated into animals in three specific brain areas, the

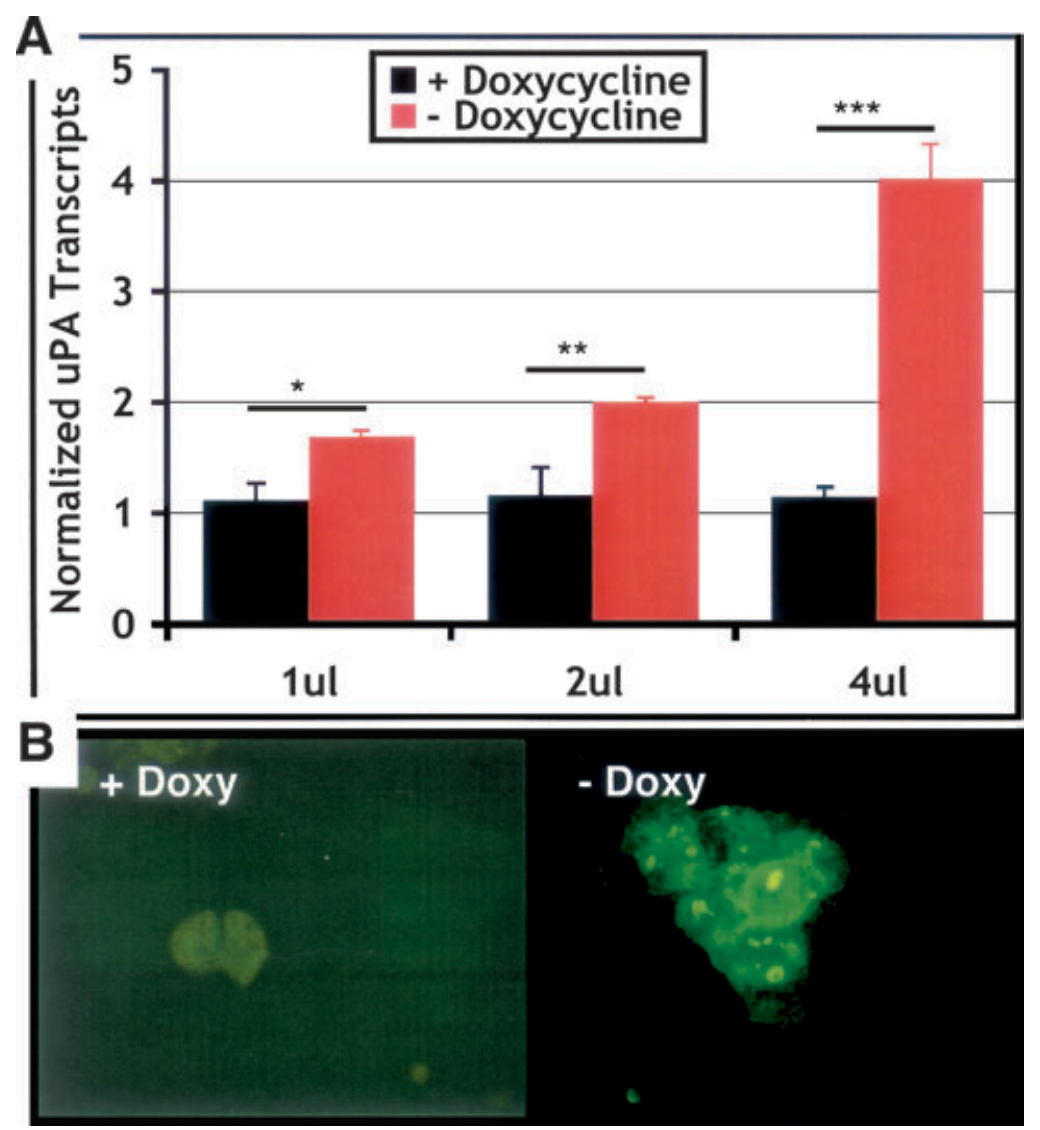

FIG. 3. In vitro expression of Lenti-uPA-His6 in HEK293T cells. (A) Quantification of uPA expression by means of qRT-PCR. Using polybrene protocol (see Methods), HEK293T cells were infected with 1, 2 or $4 \mu \mathrm{L}$ of the Lenti-uPA-His6 lentiviruses expressing uPA-His6 in culture medium with/without doxycycline $(30 \mathrm{ng} / \mathrm{mL})$. After $48 \mathrm{~h}$, culture medium was removed, one part of the cells was used for total RNA extraction, cDNA preparation and qRT-PCR using uPA-specific oligos; results were normalized against GAPDH and $\beta$-actin genes used as endogenous controls. (B) Immunocytochemistry after HEK293T cells infected with Lenti-uPA-His6. The remaining cells infected with $4 \mu \mathrm{L}$ of Lenti-uPA-His6 were used for fluorescence microscopy using a mouse histidine-tag antibody to check for uPA-His6 protein expression and regulation by doxycycline $(30 \mathrm{ng} / \mathrm{mL}) .{ }^{*} P<0.05 ; * * P<0.01 ; * * * P<0.001$. 
NAcc, the VTA or the VSub (see Methods). After 7 days of recovery under normal feeding conditions (no doxycycline), animals were administered cocaine under a chronic protocol (single daily injection of $15 \mathrm{mg} / \mathrm{kg}$ ) in the absence of doxycycline, enabling full expression of uPA (or GFP in control animals) in the infected brain area, and locomotor activity was assessed. Drug administration was performed 30 min after the animal had been placed in the behavioural cage (after the habituation period); therefore, locomotor activity measured during the initial 30 min shows the animal's activity in the absence of drug, as an internal control. As can be observed in this initial stage (Figs 46), expression of uPA, uPA-Mut or GFP did not affect this basal activity in the absence of drug. After drug administration (at $30 \mathrm{~min}$ ) a strong increase in activity was observed in all animal groups, with a peak at $40-45 \mathrm{~min}$.

Animals treated with GFP-expressing virus (control virus) displayed a maximal peak intensity of $c a .3000 \mathrm{~mm}$ at $40 \mathrm{~min}$ (distance travelled per minute, Fig. 4). By contrast, animals expressing wildtype uPA display locomotor activity peaks 10-12-fold stronger and delayed by $c a .5 \mathrm{~min}$ (Fig. 5). This delay is also observed in animals expressing the mutated uPA. This delay in maximal activity may well reflect the fact that cocaine induces uPA translation from already accumulated mRNA and that it is this increase in uPA protein that affects locomotor activity when the drug is delivered. We believe that in these repeated cocaine administration protocols uPA is translated rapidly every time after cocaine injection. Obviously the half-life of the protein has no incidence on locomotion responses because druginduced effects are very fast (within 15-60 min). In addition, the effects only occur when animals are receiving drug, as a single injection daily, before monitoring their behaviour. The intensity of the response is dependent upon the brain area. With animals expressing wild-type uPA in the NAcc, the maximal peak intensity at $45 \mathrm{~min}$ was about $29000 \mathrm{~mm}$ (Fig. 5, initial sessions without doxy); also the peak intensity increased each day, displaying a maximal activity after about 5 days of chronic drug administration. The observed behavioural changes were highest in animals overexpressing wild-type uPA in the VTA (Fig. 5), with a maximum of ca. $35000 \mathrm{~mm}$ (peak at $45 \mathrm{~min}$ at day 5), i.e. a 12.3-fold higher locomotor activity than control animals was observed under these conditions. By contrast, when uPA was expressed in the VSub of the hippocampus, a very strong increase in locomotor activity, of similar magnitude (about 12.1-fold increase over GFP-expressing control animals) was also observed (Figs 4 and 5). Two different areas of the hippocampus had been targeted, designated VSub-1 and VSub-2, based on previous data indicating that these areas regulate dopaminergic activity in the ventral tegmental areas (Legault et al., 2000; Floresco et al., 2001). Overexpression of uPA in any of these two targeted hippocampal areas induced a comparable magnitude of changes in locomotor activity.

After 5 days of chronic cocaine administration in the absence of doxycycline, all animals were fed with $0.02 \%$ doxycycline in their drinking water, enabling down-regulation of uPA and GFP expression. As shown in Fig. 4, doxycycline had no effects on control animals injected with GFP-expressing virus: no change in locomotor activity upon cocaine administration was observed in these animals during the doxycycline period, as compared with the previous days. By contrast, animals treated with virus expressing wild-type uPA- displayed very strong but rapidly decreasing locomotor activity after 4 days of doxycycline administration (Fig. 5). This drop in activity was observed for all uPA-expressing animals, irrespective to the brain area involved. This effect was followed in more detail immediately after switching the regimen (day 5, 2 to $18 \mathrm{~h}$ after regimen change) for animals expressing uPA in the NAcc and in the VTA: as shown in
Fig. 5, the drop in activity was very rapid during this initial period (day $5+2 \mathrm{~h}$ to day 6 ).

Four days later, the locomotor activity had stabilized to low levels in all groups. This stage correlates with suppression of uPA overexpression in these brain areas. Then doxycycline was removed from the regimen, enabling re-expression of wild-type uPA (or of GFP in control animals, Fig. 4) in the lentivirus-infected brain areas. As shown in Fig. 5 (second period without doxycycline), uPA re-expression induced a progressive increase in locomotor activity that again progressively reached highest levels (similar in magnitude to those observed initially at day 3 during the first period) before doxycycline addition. However, no such change was observed over that period in control animals upon re-expression of GFP (Fig. 4); locomotor activity remained unaffected throughout the experimental period in these animals.

The in vivo effects of uPA were then further evaluated using its mutated form. This should act as a dominant negative, thus interfering with endogenous uPA that is induced upon cocaine treatment. Therefore it is expected that upon expression of mutated uPA, behavioural changes would be reduced over controls (GFP-expressing animals), because the mutated uPA would compete with the endogenous protein for binding to the uPA receptor. Indeed this hypothesis was confirmed (Fig. 6): in the absence of doxycycline, locomotor activity was decreased by $40 \%$ over control animals $(2500$ vs. $4000 \mathrm{~mm} / \mathrm{min}$ ); but when doxycycline was added to drinking water, which would down-regulate lentiviral-mediated expression of the mutated uPA, while the endogenous wild-type uPA remained induced by cocaine administration, the distance travelled $(\approx 4000 \mathrm{~mm} / \mathrm{min})$ immediately increases after regimen switch and matches the levels observed with the control GFP-infected animals (Fig. 6).

To evaluate the effects of cocaine doses on locomotor activity, animals $(n=6)$ were injected in the NAcc with lenti-uPA (expressing the wild-type form of uPA) and behavioural changes were assessed. As shown in Fig. 7, the increase in locomotor activity after cocaine administration is dose dependent: in the absence of doxycycline, administration of $7 \mathrm{mg} / \mathrm{kg}$ of cocaine results in a peak of $12000 \mathrm{~mm} / \mathrm{min}$ at $45 \mathrm{~min}$, which increases dose-dependently up to $19000 \mathrm{~mm} / \mathrm{min}$ at $30 \mathrm{mg} / \mathrm{kg}$. Animals fed doxycycline also display dose-dependent changes in locomotor activity, but the levels are much lower in each case, as expected, and are similar to levels from control animals (injected with Lenti-GFP).

Immunohistochemistry confirmed that the observed behavioural changes in activity corroborate with the levels of uPA re-expression in the treated brain areas (Fig. 8). At experimental day 5, i.e. immediately before addition of doxycycline, sets of animals $(n=3)$ were killed, and the brains were removed and used for immunohistochemistry to check for the expression of UPA or GFP, whereas the rest of the animals received doxycycline and were used for further behavioural analysis. As shown in Fig. 8, full expression of uPA or GFP was maintained in all brain areas under investigation throughout this first period. This is in agreement with previous observations (Bahi et al., 2004) and the fact that lentiviruses are known to infect host cells permanently, as retroviruses incorporate their genome into the host genome. At day 9, i.e. immediately prior to removing doxycycline to the rest of the animals, a further set of animals $(n=3)$ treated with lenti-uPA was killed and gene expression was evaluated on these brains from doxycycline-fed, lenti-uPA-treated animals: immunohistochemistry revealed no significant detection of uPA in the injection sites in all brain regions (Fig. 8). Finally, at the end of the experimental period, i.e. at day 17, a set of lenti-uPA-treated animals was killed and gene expression evaluated again by means of immunohistochemistry: full gene expression of uPA was observed in the target areas (Fig. 8). 


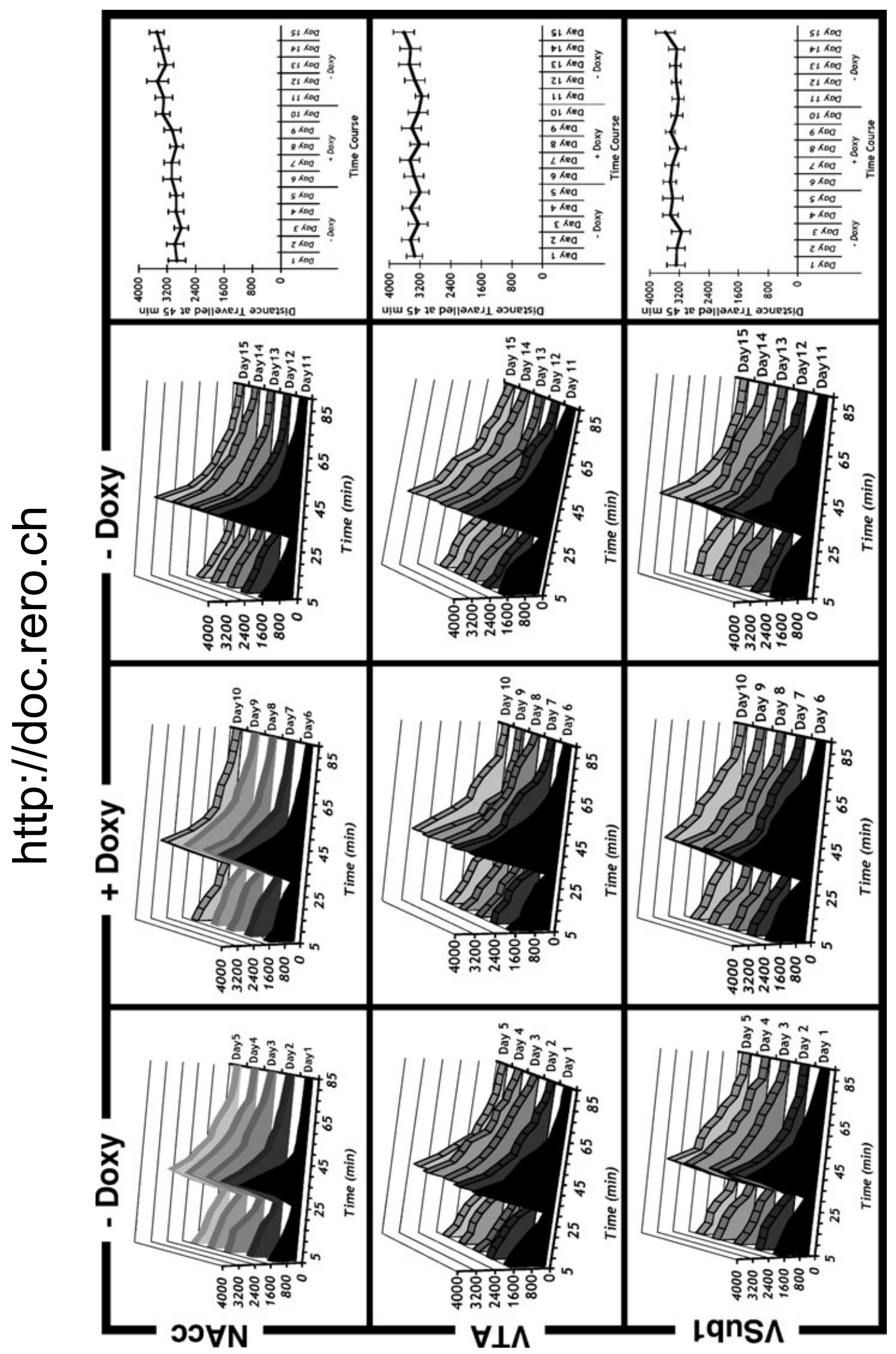

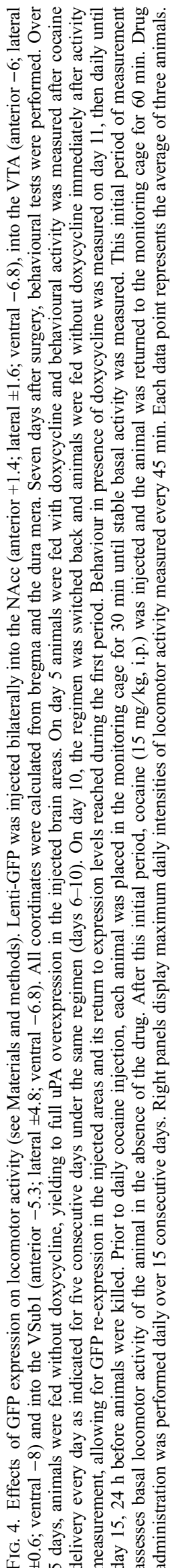




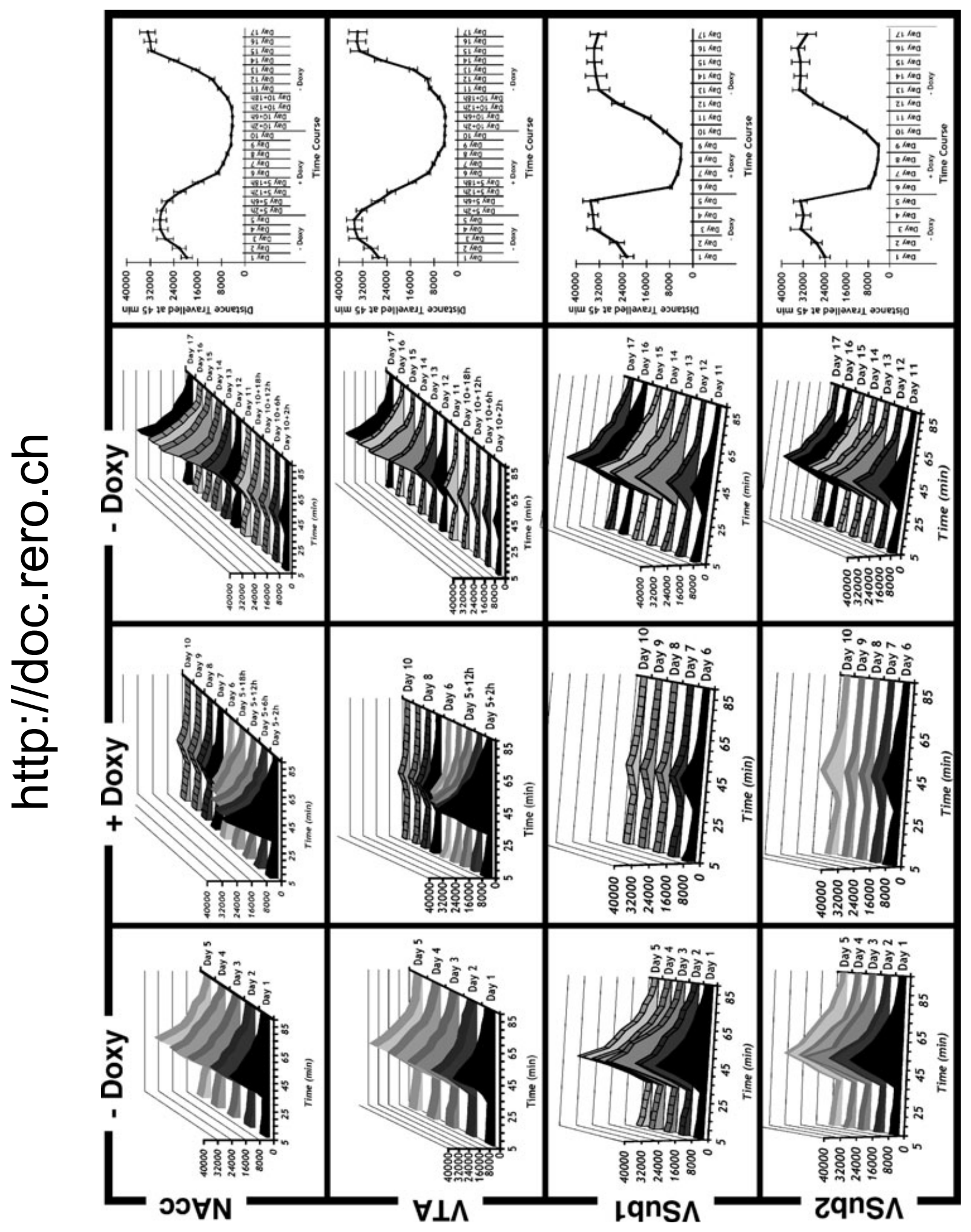

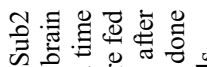

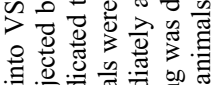

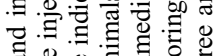

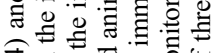

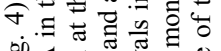

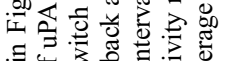

능

등 흘

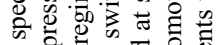

出 区.

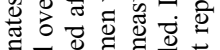

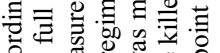

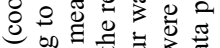

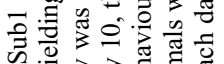

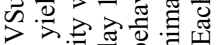

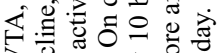

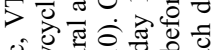

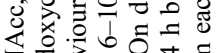

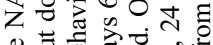

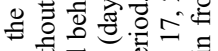

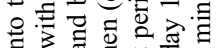

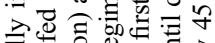

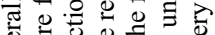

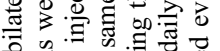

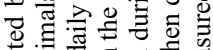

. 할

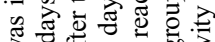

3 的可

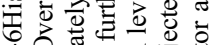

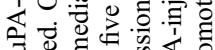

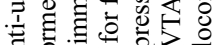

현

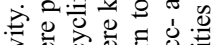

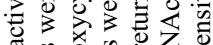

tᄒ

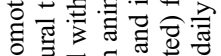

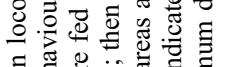

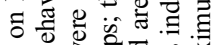

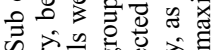

$>$ 总

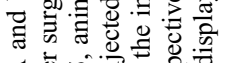

एक

$>$ 屁过

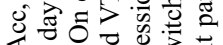

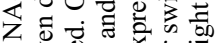

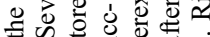

$\exists \dot{\mp}$ 징

于

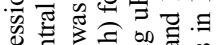

둥 $>\infty$.

ช..

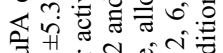

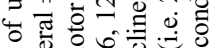

哓

我论

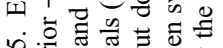

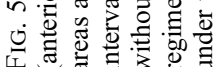




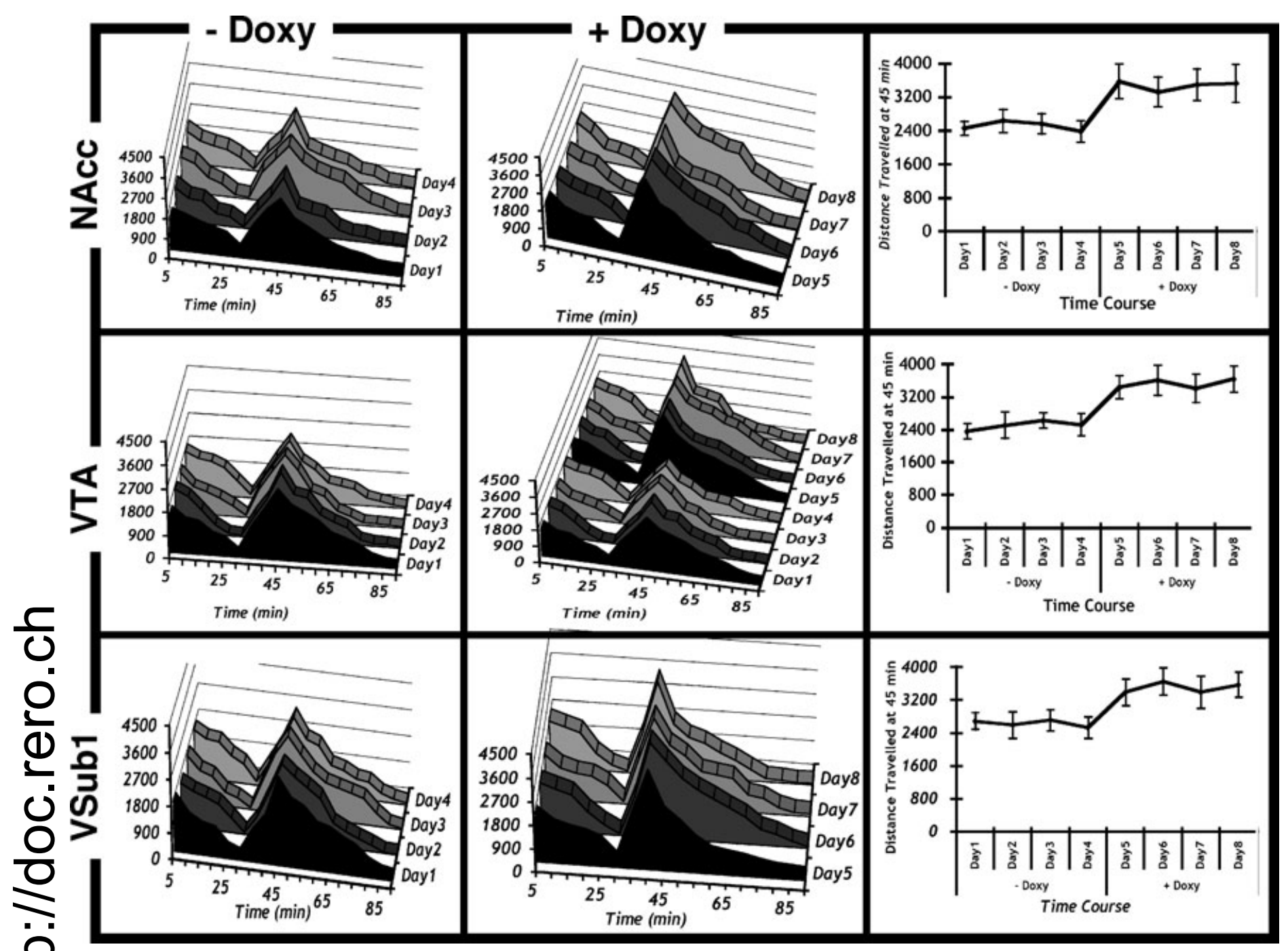

FIG. 6. Effects of mutated form of uPA expression in the NAcc, VTA and VSub on locomotor activity. Lenti-uPA-Mut was injected bilaterally into the NAcc, VTA and VSub1 in the co-ordinates specified in Fig. 4. Seven days after surgery, behavioural tests were performed. Over 5 days, animals were fed without doxycycline, yielding to full overexpression of mutated uPA in the injected brain areas. On day 5 animals were fed with doxycycline and behavioural activity was measured after cocaine delivery every day as indicated for five consecutive days under the same regimen (days 6-10). Right panels display maximum daily intensities of locomotor activity measured every $45 \mathrm{~min}$.

This demonstrates that lentivirus-mediated overexpression in the absence of doxycycline lasted throughout the experimental period. As behavioural activity was drastically changed depending upon uPA but was not affected by changes in GFP expression, these data together with the effects observed with the dominant negative form of uPA clearly show that uPA strongly modulates behavioural activity upon chronic cocaine exposure.

\section{Discussion}

This study was prompted after we identified uPA from a screening for genes up-regulated after cocaine treatment. In the present study we confirm the up-regulation of uPA in several brain regions and in different protocols of cocaine administration. Three independent approaches fully confirm cocaine-mediated uPA up-regulation: microarray screening of ca. 5000 rat genes (data not shown), microarray analysis of uPA-specific oligonucleotides under 28 different conditions (regions/protocols) and qRT-PCR of a replicate set of experiments under the same 28 different conditions, which perfectly matches previous data. Together, this firmly establishes a role of uPA in response to cocaine, an observation that was further consolidated in this study by means of monitoring behavioural changes after local injection of a regulatable uPA-expressing lentivirus. Impressive changes in locomotor activity during chronic cocaine administration were reproducibly observed: up to a 12-fold increase in correlation with uPA expression. Up-regulation was greatest in the mesolimbic dopaminergic pathway, a brain region efficiently activated upon administration of drugs of misuse. Such drugs involve profound synaptic rearrangements and plasticity, as a basic mechanism for reinforcement. The regulatable lentivirus system developed for this study enables efficient switch in local gene expression, which induces a parallel switch in locomotor activity in a highly reproducible way.

In this study we found that cocaine treatment enhances uPA gene expression and that uPA overexpression further augments the influence of cocaine on animal behaviour. Surprisingly, uPA up-regulation has no effect without cocaine. This strongly suggests that uPA constitutes a limiting factor in the capacity of cocaine to stimulate 


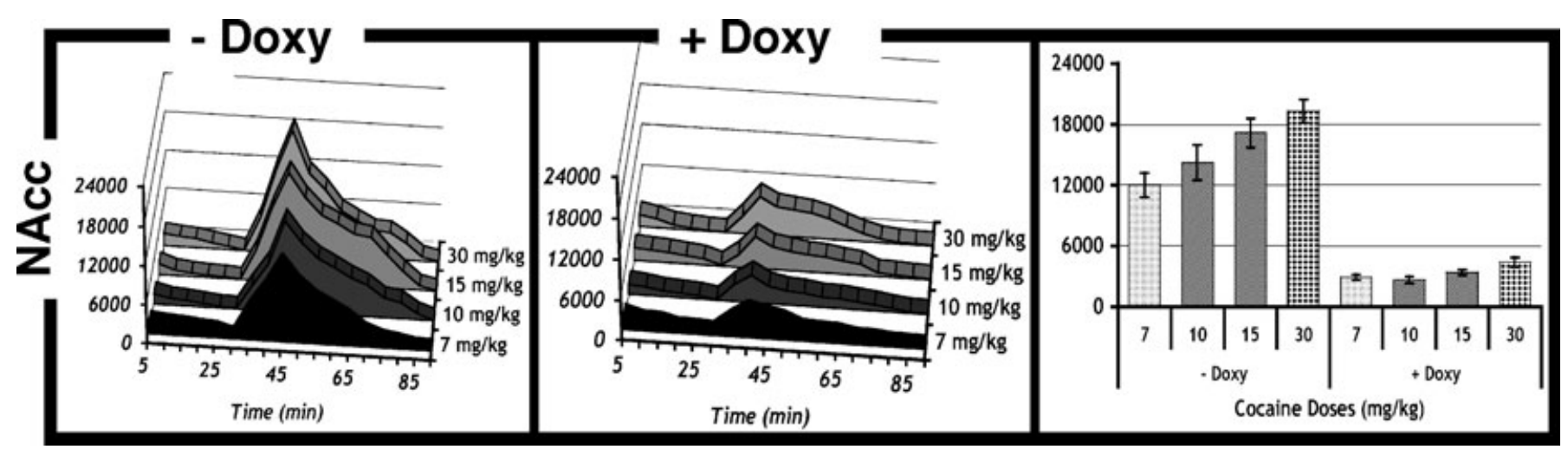

FIG. 7. Effects of cocaine doses and uPA expression in the NAcc on locomotor activity. Lenti-uPA-6His was injected bilaterally into the NAcc using the co-ordinates specified in Fig. 4. Seven days after operation, behavioural tests were performed. Over 5 days, animals are fed without doxycycline, yielding to full overexpression of uPA in the injected brain area and locomotor activity was monitored after cocaine injection. Each group was injected with different doses of cocaine: 7, 10, 15 or $30 \mathrm{mg} / \mathrm{kg}$. On day 5 animals were fed with doxycycline and the behavioural activity was measured using the same drug regimen delivery as in the first period. Right panel displays maximum daily intensities of the locomotor activity measured every 45 min. Each data point represents the average of three animals.

locomotor activity. The mechanisms remains to be further elucidated, but some hypotheses may be discussed.

\section{Reversible nature of the UPA effect - groups of genes induced after cocaine and mechanisms}

The reversible nature of the uPA effect, as shown in Figs 4-6, indicates that in order to be effective, uPA should be constantly present. This raises the possibility that uPA exerts its effect through an aproteolytically independent mechanism, e.g. attachment-detachment by means of its interaction with its receptor uPAR. The role of uPA in the brain for plasmin activation is not clear. However, a recent study has shown that the tissue plasminogen activator-plasmin system participates in the rewarding effect of morphine by regulating dopamine release (Nagai et al., 2004). This finding fully correlates with the observation described in this paper with cocaine. Together, this indicates that the plasmin system may play a central role in druginduced plasticity.

Plasminogen production in brain is at least $10^{3}$-fold lower than in liver and thus uPA-mediated proteolytic cascade is unlikely to be of major significance (Davies et al., 1998). Upon binding to its receptor, the UPA-uPAR complex serves as co-receptor for various integrins and may directly bind to the matrix-associated integrin-ligand vitronectin. Such uPAR-dependent interactions are relevant for synaptic rearrangement, spine morphology, long-term potentiation and also for kindling formation in adult brain (Yoshida \& Shiosaka, 1999). The interaction can be blocked by the plasminogen activator inhibitor-1 (PAI-1), a uPA endogenous inhibitor (Zhou et al., 2003). A balanced level of uPA and PAI-1 is required alternately to disrupt and re-form interactions of vitronectin with UPAR and regulate integrin signalling. Therefore, drug-induced uPA expression may be implied in regulation of integrin signalling. This hypothesis supports observations describing drug-mediated induction of other surface proteins, which also act through integrins and mediate synaptic plasticity. For example, the integrin-binding tetraspannin CD81 (Brenz-Verca et al., 2001; Michna et al., 2002; Bahi et al., 2004) is strongly induced by cocaine. Furthermore, Ephrin-B1 and Ephrin-A, which both regulate integrin function (Davy \& Robbins, 2000), and also several semaphorins with integrin-binding motifs, such as Sema3C, Sema4G and Sema7A (Pasterkamp et al., 2003), are 4-12-fold regulated upon cocaine (Bahi \& Dreyer, 2004).

\section{Role of hippocampal activation on accumbal dopamine}

In this study we have shown that local uPA overexpression within the dopaminergic mesolimbic pathway induces very drastic changes in behaviour after cocaine administration, resulting in an up to 12 -fold increase in locomotor activity. The effect was very strong when the gene was expressed into the VTA directly. But an effect of similar intensity was reached when the gene was expressed into the ventral subiculum region of the hippocampus. This agrees with our microarray data, which showed that all protocols of cocaine administration induced a strong up-regulation in the hippocampus, similar to the up-regulation observed in mesolimbic dopaminergic areas.

Several studies have reported that the ventral hippocampus elevates nucleus accumbens dopamine by activating dopaminergic neurons of the ventral tegmental area (Blaha et al., 1997; Legault et al., 2000; Floresco et al., 2001). The nucleus accumbens is a central component of basal ganglia and integrates signals arising from cortical and limbic areas to modulate motor output (Mogenson et al., 1993). Dopaminergic projections from the ventral tegmental area to the nucleus accumbens septi are involved in investigatory behaviours evoked by novel stimuli. They are related to the reinforcement of adaptive investigatory approaches evoked by natural rewards or by rewards invoked by habit-forming drugs and drugs of misuse (Wise \& Rompré, 1989; Wise, 1996; Schultz, 1998). Reinforcement and environmental memory play key roles in addiction and many studies have stressed the role of the hippocampus in relation to the action of drugs of misuse, by modulating dopaminergic transmission and increasing accumbal dopamine through glutamatergic impulse on the VTA (Floresco et al., 2001; Legault et al., 2000). Excitatory glutamatergic inputs to the nucleus accumbens arise from ventral subiculum spiny neurons, which are in close apposition to dopaminergic projections arising from the ventral tegmental area (Legault et al., 2000). There are several projection pathways that can potentially mediate subicular regulation of dopaminergic cell firing (see, for example, Floresco et al., 2001, for a discussion) and therefore several subsites of the subiculum may act in activating dopaminergic neurons on the ventral tegmental area. For this reason, we have targeted two different subicular regions with our regulatable lentivirus, choosing coordinates VSub-1 and VSub-2 from other studies (Legault et al., 2000; Floresco et al., 2001). Our in vivo study fully confirms their data and further establishes the role of hippocamal stimulation in very efficiently modulating addiction-related behaviours, at 


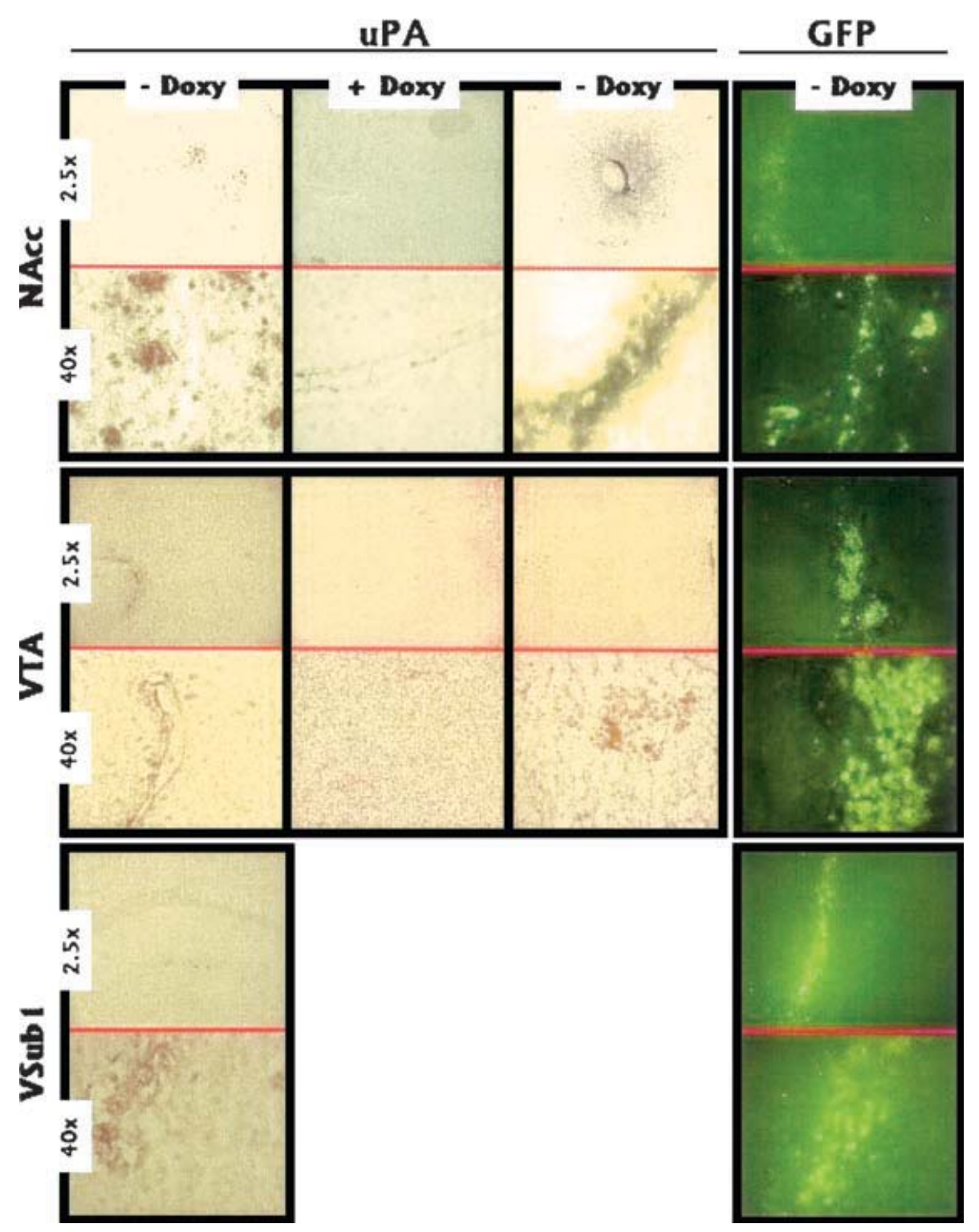

FIG. 8. Immunohistochemistry of uPA and GFP expression in the NAcc, VTA and VSub after in vivo transfer of either lenti-uPA-6His or lenti-GFP. For uPA expression: $2 \mu \mathrm{L}$ of lenti-uPA-6His was injected into the NAcc, VTA and VSub according to the coordinates described in the Methods section. Animals were given either 5\% sucrose or 5\% sucrose $/ 0.02 \%$ doxycycline. Animals were killed at the end of each period (at day 5 , before addition of doxycycline for NAcc and VTA injected groups) '-Doxy', at day 9 (before removal of doxycycline for NAcc and VTA injected groups) '+ Doxy' and at day 17 (day 15 for animals injected in the VSub) '-Doxy'. For GFP expression (Control animals): $2 \mu \mathrm{L}$ of lenti-GFP was injected into the NAcc, VTA and VSub according to the coordinates described in the Methods section. Animals were killed in the end of the second doxycycline-free period (-Doxy). All brains were dissected out, and processed for immunohistochemistry (see Materials and methods ). For each region and period; upper panels: magnification $\times 2.5$, lower panels: magnification $\times 40$.

magnitudes similar to those reached by direct stimulation of the nucleus accumbens dopamine release.

\section{Implication for addiction}

The neural pathways involved in addiction are still poorly known and the role of cortical and hippocampal pathways in mediating reinforcement are still under investigation. Dysfunction of the dopaminergic system has long been implicated as a primary factor in the pathophysiology of addiction, which involves strong plasticity of the mesolimbic dopaminergic pathway. A role of extracellular proteases in synaptic rearrangement is now well established. From the present study, uPA is clearly induced upon chronic drug intake, and, because of its known implication in excitotoxicity (Masos \& Miskin, 1997; Kishi et al., 1999), a role of uPA in drug-induced epileptogenesis may be possible. On the other hand, drug-mediated uPA expression may also promote neuronal death, associated in some cases with chronic drug intake, by means of plasmin-catalysed degradation of laminin (Chen \& Strickland, 1997). Furthermore, our data show that uPA is a very strong potential candidate in modulating mesolimbic dopaminergic pathways and alteration of neural circuits crucial to addiction-related processes. The effects are specific because, for example, GFP overexpression does not show any such effect, and they are reversible because decreasing uPA expression in specific brain areas restores drug-related behaviour almost back to normal. To clarify these effects, the doxycycline-regulatable lentivirus used in the 
present study proved to be an ideal tool for assessing the role of uPA in vivo and its putative function in addiction.

\section{Acknowledgements}

This research was supported by a Swiss National Foundation grant 3100059350 and 3100AO-100686 (J.L.D.) and NIH grant NIDDK RO1 DK5870203 (T.K.). We are also very grateful to Mrs C. Deforel-Poncet for their skilful assistance.

\section{Abbreviations}

Cpu, caudate putamen; HEK293T, human embryonic kidney 293T cells; Hipp, hippocampus; NAcc, nucleus accumbens; qRT-PCR, quantitative real-time PCR; RT, room temperature; SNr, substantia nigra; uPA, urokinase plasminogen-type activator; VTA, ventral tegmental area.

\section{References}

Appella, E., Robinson, E.A., Ullrich, S.J., Stoppellin, M.P., Corti, A., Cassani, G. \& Blasi, F. (1981) The receptor-binding sequence of urokinase. A biological function for the growth-factor module of proteases. J. Biol. Chem., 262, 44374440.

Arai, Y., Kubota, T., Nakagawa, T., Kabuto, M., Sato, K. \& Kobayashi, H. (1998) Production of urokinase-type plasminogen activator (u-PA) and plasminogen activator inhibitor-1 (PAI-1) in human brain tumours. Acta Neurochir., 140, 377-386.

Bahi, A., Boyer, F., Kafri, T. \& Dreyer, J.L. (2004) CD81-induced behavioural changes after chronic cocaine administration. In vivo gene delivery with regulatable lentivirus. Eur. J. Neurosci., 19, 1621-1633.

Bahi, A. \& Dreyer, J.L. (2004) Cocaine-induced expression changes of axon. Guidance molecules in the adult rat brain. Mol. Cell Neurosci., in press.

Blaha, C.D., Yang, C.R., Floresco, S.B., Barr, A.M. \& Phillips, A.G. (1997) Stimulation of the ventral subiculum of the hippocampus evokes glutamate receptor-mediated changes in dopamine efflux in the rat nucleus accumbens. Eur. J. Neurosci., 16, 902-911.

Brenz-Verca, M.S., Widmer, D.A.J., Wagner, G.C. \& Dreyer, J.L. (2001) Cocaine-induced expression of the tetraspanin CD81 and its relation to hypothalamic function. Mol. Cell Neurosci., 17, 303-316.

Chan, S.L. \& Mattson, M.P. (1999) Caspase and calpain substrates: roles in synaptic plasticity and cell death. J. Neurosci. Res., 58, 167-190.

Chen, Z.L. \& Strickland, S. (1997) Neuronal death in the hippocampus is promoted by plasmin-catalyzed degradation of laminin. Cell, 91, 917925.

Davies, B.J., Pickard, B.S., Steel, M., Morris, R.G.M. \& Lathe, R. (1998) Serine proteases in rodent hippocampus. J. Biol. Chem., 273, 2300423011.

Davy, A. \& Robbins, S.M. (2000) Ephrin-A5 modulates cell adhesion and morphology in an integrin-dependent manner. EMBO J., 19, 5396-5405.

Degryse, B., Orlando, S., Resnati, M., Rabbani, S.A. \& Blasi, F. (2001) Urokinase/urokinase receptor and vitronectin/ $\alpha 2 \beta 3$ integrin induce chemotaxis and cytoskeleton reorganization through different signaling pathways. Oncogene, 20, 2032-2043.

Del Bigio, M.R., Hosain, S. \& Altumbabic, M. (1999) Localization of urokinase-type plasminogen activator, its receptor, and inhibitors in mouse forebrain during postnatal development. Int. J. Dev. Neurosci., 17, 387399.

Floresco, S.T., Todd, C.L. \& Grace, A.A. (2001) Glutamatergic afferents from the hippocampus to the nucleus accumbens regulate activity of ventral tegmental area dopamine neurons. J. Neurosci., 21, 4915-4922.

Gingrich, M.B. \& Traynelis, S.F. (2000) Serine proteases and brain damage - is there a link? Trends Neurosci., 23, 399-407.

Gottschalk, P.E. \& Deb, S. (1996) Regulation of matrix metalloproteinase expressions in astrocytes, microglia and neurons. Neuroimmunomodulation, 3, 69-75.

Hedou, G., Jongen-Relo, A.L., Murphy, C.A., Heidbreder, C.A. \& Feldon, J. (2002) Sensitized Fos expression in subterritories of the rat medial prefrontal cortex and nucleus accumbens following amphetamine sensitization as revealed by stereology. Brain Res., 950, 165-179.

Heidbreder, C., Thomson, A.C. \& Shippenberg, T.S. (1996) Role of extracellular dopamine in the initiation and long-term expression of behavioural sensitization to cocaine. J. Pharmacol. Exp. Ther, 276, 490-502.
Kacharmina, J.E., Crino, P.B. \& Eberwine, J. (1999) Preparation of cDNA from single cells and subcellular regions. Meth. Enzymol., 303, 3-18.

Kishi, T., Kato, M., Shimizu, T., Kato, K., Matsumoto, K., Yoshida, S., Shiosaka, S. \& Hakoshima, T. (1999) Crystal structure of neuropsin, a hippocampal protease involved in kindling epileptogenesis. J. Biol. Chem., 274, 4220-4224.

Komai, S., Matsuyama, T., Matsumoto, K., Kato, K., Kobayashi, M., Imamura, K., Yoshida, S., Ugawa, S. \& Shiosaka, S. (2000) Neuropsin regulates an early phase of Schaffer-collateral long-term potentiation in the murine Hippocampus. Eur. J. Neurosci., 12, 1479-1486.

Legault, M., Romprè, P.P. \& Wise, R.A. (2000) Chemical stimulation of the ventral hippocampus elevates nucleus accumbens dopamine by activating dopaminergic neurons of the ventral tegmental area. J. Neurosci., 20, 16351642.

Lüthi, A., Van der Putten, H., Botteri, F.M., Mansuy, I.M., Meins, M., Frey, U., Sansig, G., Portet, C., Schmutz, M., Schroder, M., Nitsch, C., Laurent, J.P. \& Monard, D. (1997) Endogenous serine protease inhibitor modulates epileptic activity and hippocampal long-term potentiation. J. Neurosci., 17, 46884699.

Masos, T. \& Miskin, R. (1996) Localisation of urokinase-type plasminogen activator mRNA in the adult mouse brain. Mol. Brain Res., 35, 139-148.

Masos, T. \& Miskin, R. (1997) mRNA encoding urokinase-type plasminogen activator and plasminogen activator inhibitor-1 are elevated in the mouse brain following kainate-mediated excitation. Mol. Brain Res., 47, $157-169$.

Michna, L., Brenz-Verca, M., Dreyer, J.L. \& Wagner, G.C. (2002) Methods to examine molecular changes and behavioural effects of drug administration. Brain Res. Protocols, 9, 181-196.

Miskin, R. \& Masos, T. (1997) Transgenic mice overexpressing urokinase-type plasminogen activator in the brain exhibit reduced food consumption, body weight and size and increased longetivity. J. Gerontol. Biol. Sci., 52A, B118B124.

Mogenson, G.J., Brudzynski, S.M., Wu, M., Yang, C.Y. \& Yim, C.C.Y. (1993) From motivation to action: a review of dopaminergic regulation of limbic to nucleus accumbens to ventral apllidum to pedunculopontine nucleus circuitries involved in limbic-motor integration. In Kallivas, P.W. \& Barnes, C.D. (Eds), Limbic Motor Circuits in Neuropsychiatry. CRC, Boca Raton, FL, pp. 193-236.

Mühlbauer, M., Allard, B., Bosserhoff, A.K., Kiessling, S., Herfarth, H., Rogler, G., Schölmerich, J., Jobin, C. \& Hellerbrand, C. (2004) Differential effects of deoxycholic acid and taurodeoxycholic acid on NF kappa $\}$ B signal transduction and il-8 gene expression in colonic epithelial cells. Am. J. Physiol. Gastrointest. Liver Physiol., 286, G1000-G1008.

Mustjoki, S. (2003) Urokinase, urokinase receptor and ICAMS in human leukemia. Academic dissertation. Department of Virology, Haartman Institute, University of Helsinki, Finland.

Nagai, T., Yamada, K., Yoshimura, M., Ishikawa, K., Miyamoto, Y., Hashimoto, K., Noda, Y., Nitta, A. \& Nabeshima, T. (2004) The tissue plasminogen activatorñplasmin system participates in the rewarding effect of morphine by regulating dopamine release. Proc. Natl Acad. Sci. USA, 101, $3650-3655$.

Naldini, L., Blomer, U., Gallay, P., Ory, D., Mulligan, R., Gage, F.H., Verma, I.M. \& Trono, D. (1996) In vivo gene delivery and stable transduction of nondividing cells by a lentiviral vector. Science, 272, 263-267.

Nestler, E. (2000) Genes and addiction. Nature Genet., 26, 277-281.

Nienaber, V., Wang, J., Davidson, D. \& Henkin, J. (2000) Re-engineering of human urokinase provides a system for structure-based drug design at high resolution and reveals a novel structural subsite. J. Biol. Chem., 275, 72397248.

Pasterkamp, R.J., Peschon, J.J., Spriggs, M.K. \& Kolodkin, A.L. (2003) Semaphorin 7A promotes axon outgrowth through integrins and MAPKs. Nature, 424, 398-405.

Rosenberg, G.A., Cunningham, L.A., Wallace, J., Alexander, S., Estrada, E.Y., Grossetete, M., Razhagi, A., Miller, K. \& Gearing, A. (2001) Immunohistochemistry of matrix metalloproteinases in reperfusion injury to rat brain: activation of MMP-9 linked to stromelysin-1 and microglia in cell cultures. Brain Res., 893, 104-112.

Schultz, W. (1998) Predictive reward signal in dopaminergic neurons. J. Neurophysiol., 80, 1-27.

Schwab, J.M., Weyermann, R. \& Schluesener, H.J. (2001) Serine proteases and brain damage - Contribution of the urokinase-type plasminogen activator system. Trends Neurosci., 24, 8-9.

Shalon, D., Smith, S.J. \& Brown, P.O. (1996) A DNA microarray system for analyzing complex DNA samples using two-color fluorescent probe hybridization. Genome Res., 6, 639-645. 
Shimizu, C., Yoshida, S., Shibata, M., Kato, K., Momota, Y., Kazumasa Matsumoto, K., Shiosaka, T., Midorikawa, R., Kamachi, T., Kawabe, A. \& Shiosaka, S. (1998) Characterization of recombinant and brain neuropsin, a plasticity-related serine protease. J. Biol. Chem., 273, 11189-11196.

Shiosaka, S. \& Yoshida, S. (2000) Synaptic microenvironment-structural plasticity, adhesion molecules, proteases and their inhibitors (Review). Neurosci. Res., 37, 85-89.

Sokabe, T., Yamamoto, K., Ohura, N., Nakatsuka, H., Qin, K., Obi, S., Kamiya, A. \& Ando, J. (2004) Differential regulation of urokinase-type plasminogen activator expression by fluid shear stress in human coronary artery endothelial cells. Am. J. Physiol. Heart Circ. Physiol., 287, H2027-H2034.

Stepanova, V., Jerke, U., Sagach, V., Lindschau, C., Dietz, R., Haller, H. \& Dumler, I. (2002) Urokinase-dependent human vascular smooth muscle cell adhesion requires selective vitronectin phosphorylation by ecto-protein kinase CK2. J. Biol. Chem., 277, 10265-10272.

Sumi, Y., Dent, M.A., Owen, D.E., Seeley, P.J. \& Morris, R.J. (1992) The expression of tissue and urokinase-type plasminogen activators in neural development suggests different modes of proteolytic involvement in neuronal growth. Development, 116, 625-637.
Wang, G.J., Collinge, M., Blasi, F., Pardi, R. \& Bender, J.R. (1998) Posttranscriptional regulation of urokinase plasminogen activator receptor messenger RNA levels by leukocyte integrin engagement. Proc. Natl Acad. Sci. USA, 95, 6296-6301.

Wang, Y., Dang, J., Wang, H., Allgayer, H., Murrell, G.A.C. \& Boyd, D. (2000) Identification of a novel nuclear factor-kappaB sequence involved in expression of urokinase-type plasminogen activator receptor. Eur. J. Biochem., 267, 3248-3254.

Wise, R.A. \& Romprè, P.P. (1989) Brain dopamine and reward. Ann. Rev. Psychol., 40, 191-225.

Wise, R.A. (1996) Neurobiology of addiction. Curr. Opin. Neurobiol., 6, $243-$ 251.

Yong, V.W., Krekoski, C.A., Forsyth, P.A., Bell, R. \& Edwards, D.R. (1998) Matrix metalloproteinases and diseases of the CNS. Trends Neurosci., 21, 75-80.

Yoshida, S. \& Shiosaka, S. (1999) Plasticity-related serine proteases in the brain. Int. J. Mol. Med., 3, 405-409.

Zhou, A., Huntington, J.A., Pannu, N.S., Carrell, R.W. \& Read, R.J. (2003) How vitronectin binds PAI-1 to modulate fibrinolysis and cell migration. Nature Struct. Biol., 10, 541-544. 